\title{
Noise-Induced Synchronization and Antiresonance in Interacting Excitable Systems: Applications to Deep Brain Stimulation in Parkinson's Disease
}

\author{
Jonathan D. Touboul,,$^{1, *}$ Charlotte Piette, ${ }^{1,2}$ Laurent Venance, ${ }^{2}$ and G. Bard Ermentrout ${ }^{3}$ \\ ${ }^{1}$ Department of Mathematics and Volen National Center for Complex Systems, \\ Brandeis University, Waltham, Massachusetts 02453, USA \\ ${ }^{2}$ Center for Interdisciplinary Research in Biology (CIRB), College de France, CNRS, INSERM, \\ PSL Research University, Paris 75005, France \\ ${ }^{3}$ Department of Mathematics, University of Pittsburgh, Pittsburgh, Pennsylvania 15213, USA
}

(Received 23 May 2019; revised manuscript received 17 January 2020; accepted 12 February 2020; published 27 March 2020)

\begin{abstract}
We study the nonlinear dynamics of a surprising phenomenon arising in large networks of excitable elements in response to noise: while at low noise, solutions remain in the vicinity of the resting state and large-noise solutions show asynchronous activity, the network displays orderly, perfectly synchronized periodic responses at intermediate levels of noise. This noise-induced synchronization, distinct from classical stochastic resonance, is fundamentally collective in nature. Indeed, we show that, for noise and coupling within specific ranges, an asymmetry in the transition rates between a resting and an excited regime progressively builds up, leading to an increase in the fraction of excited neurons eventually triggering a chain reaction associated with a macroscopic synchronized excursion and a collective return to rest where this process starts afresh, thus yielding the observed periodic synchronized oscillations. We further uncover a novel antiresonance phenomenon in this regime: noise-induced synchronized oscillations disappear when the system is driven by periodic stimulation with frequency within a specific range (high relative to the spontaneous activity). In that antiresonance regime, the system is optimal for measures of information transmission. This observation provides a new hypothesis accounting for the efficiency of high-frequency stimulation therapies, known as deep brain stimulation, in Parkinson's disease, a neurodegenerative disease characterized by an increased synchronization of brain motor circuits. We further discuss the universality of these phenomena in the class of stochastic networks of excitable elements with specific coupling and illustrate this universality by analyzing various classical models of neuronal networks. Altogether, these results uncover some universal mechanisms supporting a regularizing impact of noise in excitable systems, reveal a novel antiresonance phenomenon in these systems, and propose a new hypothesis for the efficiency of high-frequency stimulation in Parkinson's disease.
\end{abstract}

DOI: 10.1103/PhysRevX.10.011073

Subject Areas: Biological Physics, Complex Systems, Nonlinear Dynamics

\section{INTRODUCTION}

Coupled systems of excitable elements subject to noise are commonly used to model natural and physical phenomena. They describe, in particular, laser emission [1], chemical reactions where noise reportedly supports traveling waves [2], climate dynamics [3], cardiac tissue and other physiological processes [4], gene networks where excitability in the presence of noise was suggested as a possible mechanism for transient cellular differentiation

jtouboul@brandeis.edu

Published by the American Physical Society under the terms of the Creative Commons Attribution 4.0 International license. Further distribution of this work must maintain attribution to the author(s) and the published article's title, journal citation, and DOI.
[5], and neurons and ion channels [6] (see Ref. [7] for a review).

Excitable systems have in common the existence of a rest state, a globally attractive fixed point when the system is unperturbed, and two typical responses to perturbations: small perturbations result in small amplitude responses, while sufficiently strong perturbations (bringing the system to cross a quasiseparatrix) lead to a long excursion (a spike) through an excited state, followed by a return to rest after a refractory period during which the system essentially cannot be excited. Single excitable elements subject to noise have been widely studied theoretically and experimentally, and various noise-induced phenomena such as coherence resonance or self-induced stochastic resonance (SISR) were identified [7]. The latter phenomenon is associated with maximally coherent responses of a single excitable unit in response to small noise, as reported 
and thoroughly described for the FitzHugh-Nagumo model in Refs. [8-10]. A distinct type of stochastic resonance was reported in systems driven by oscillatory signals, whereby addition of a certain amount of noise enhances the coherence of the response [11]. This type of resonance has been evoked in various natural phenomena particularly in neural systems [12-14]. How these phenomena scale up in large networks of excitable elements and with larger noise levels compatible with typical fluctuations in natural systems remains a largely open problem.

Synchronized oscillations constitute a significant macroscopic state in networks of excitable systems. In brain, for instance, rhythmic macroscopic activity (a hallmark of collective neuronal synchronization) was observed in a variety of species, in various brain areas, and across a wide range of frequencies [15], and, in mammals, is reportedly related to various cognitive processes such as memory, attention, and sleep [16]. Impairments in synchronous activity are also observed in several pathologies: abnormally high synchrony is reported in epilepsy or Parkinson's disease, low synchrony in Alzheimer's disease, and altered oscillatory patterns in schizophrenia [17]. These regular behaviors emerge despite the presence of multifarious noisy fluctuations (see, e.g., Ref. [18] for a review of sources of noise in the brain) that often have a significant impact on the dynamics. Important progress has been made to understand the synchronization of oscillators (dynamical systems that oscillate intrinsically), in particular, in the frame of the Kuramoto model. An abundant literature has characterized how these oscillators can generate coherent behaviors in the presence of noise, heterogeneity in the intrinsic frequencies, graph structure of interactions [19-21], and even, particularly relevant in the present context, how noise and periodic input can shape synchronization of nonidentical oscillators [22]. Excitable systems, such as some neurons, are not intrinsic oscillators: in the absence of input, they stabilize at a fixed point, and thus synchronization of excitable elements likely relies on mechanisms in large part distinct from those of coupled oscillators.

This paper investigates a surprising and somewhat paradoxical regularizing impact of noise in large-scale networks of excitable elements: as noise is progressively increased, the network shows a sudden transition from stationary, low-amplitude fluctuations around the rest state (clamped regime) to a massive synchronization of neurons yielding coherent, high-amplitude, and periodic macroscopic oscillations (noise-induced oscillations regime) that progressively desynchronize as noise is further increased until an aynchronous regime is reached where randomness overwhelms collective and nonlinear effects. This phenomenon was observed quite early in the study of cooperative excitable systems described as active rotators $[23,24]$. Based on numerical simulations, it was shown that these systems can support stationary or time-periodic regimes for appropriate levels of noise and coupling strength, and that phase transitions between these regimes arise as a function of coupling strength and noise level: for too large noise or too strong coupling, stationary dynamics arise, yet within an appropriate range of coupling and noise levels, periodic regimes emerge, potentially where the noiseless system shows nonperiodic dynamics [23]. As pointed out later by Pham et al. [25], noise in these systems acts in two antagonistic ways, first making oscillations more regular at sufficiently low levels, and then deteriorating this regularity for stronger amplitudes. Going further, Shinomoto and Kuramoto [23] described, in coupled active rotators, the presence of two distinct transitions delineating the region of noise-induced synchrony: for small noise, a progressive increase in oscillation frequency, and, when oscillations disappear for larger noise, a sudden, discontinuous frequency drop, that the authors associate to the presence of saddle-node or Hopf transitions, later elucidated more finely by studying the dynamics of the first two Fourier modes of the phases distribution [24]. In the domain of probability theory, the possibility of the emergence of oscillations due to noise in abstract diffusion models with Gaussian solutions [26,27] or in a specific neuronal network model [28] was demonstrated through the study of the associated moment equations. Recently, the question of noise-induced oscillations has been the topic of renewed interest in applied mathematics [29,30] or physics [31,32], and different theoretical techniques on the limit equations were used to characterize these oscillations, including, in particular, slow-fast analysis, invariant manifolds, or Hopf-Cole transforms. Among numerous other important works in the domain, we shall highlight the work of Zaks and collaborators on the dynamics of coupled FitzHugh-Nagumo neurons in small noise and high excitability regimes (resting state lying in the vicinity of the excitability quasiseparatrix). Using a moment expansion and closure based on the assumption that solutions are approximately Gaussian [7,33], the authors showed how noise was indeed controlling the existence of periodic solutions, along similar lines as the study of Kurrer and Schulten on active rotators [34]. In that paper, using also a Gaussian approximation of solutions, the authors derived expressions for noise levels associated with a collective escape from a stationary point, key for the generation of oscillations. Similar to what was observed in Refs. [23,24], they further showed that low- and high-noise transitions around the periodic solutions regime were of distinct nature. However, Gaussian approximations generally do not extend beyond small noise; as noise is increased, the Gaussian approximation breaks down, and we will exhibit the emergence of a bimodal distribution crucial in the generation of noise-induced oscillations for larger noise levels. Beyond neuronal models and active rotators, similar phenomena were exhibited in hair bundles coupled with elastic forces [35] or more abstract cellular automata [36]. 
In the latter model, coupled elements can sit in one of three states (akin to rest, excited, and recovering) with exponential transitions from rest to excited (depending on the state of the system) and nonexponential transitions between the other states (transitions taking a fixed typical time), highlighting an important role of the asymmetry in transitions from rest to the excited state (stochastic and memoryless) compared to the reciprocal (delayed) transition.

Altogether, despite this ample literature, little remains known about the microscopic (i.e., single-elements) dynamics supporting noise-induced oscillations in excitable systems, and progress in numerical or mathematical analyses of mean-field equations will not allow for addressing those mechanisms. We develop here a fine scale analysis of individual trajectories, in a stochastic electrically coupled FitzHugh-Nagumo network, to unravel the microscopic mechanisms at play. Electrical coupling was indeed shown to favor the emergence of synchronized activity in biological neural networks [37-41]; it is also a particularly simple mathematical model allowing an indepth study. This analysis will highlight the respective roles of coupling levels, noise intensity, and excitability in the emergence of oscillations due to noise. Going further, the identification of these mechanisms will lead us to uncover a novel phenomenon of coupled excitable systems in the noise-induced synchronization regime. We will show that periodic forcing of the system at high frequency (relative to the spontaneous noise-induced oscillation frequency) can prevent synchronization, and set the system in a regime where it is able to maximize information transmission capabilities. Our analysis of the mechanisms leading to synchronization in turn will allow us to identify the critical requirements underlying these behaviors, highlight their universality for excitable networks with confining interactions, and conjecture the type of transition to synchrony occurring as noise or connectivity is varied.

These phenomena may have multiple applications. We particularly explore here their implications in the context of Parkinson's disease and its treatment, and will use vocabulary and concepts from neuroscience throughout the paper. Parkinson's disease is a neurodegenerative disorder classically associated with dramatic motor and cognitive symptoms, and with a pathological modification of patterns of oscillations in the basal ganglia or motor cortex [43,44]. Among the variety of factors contributing to these oscillations, studies invoked an elevated excitability of neurons [45-47] and increased electrical coupling $[48,49]$, two elements that we will see are important in the emergence of noise-induced oscillations in our models. Deep brain stimulation (DBS), an efficient symptomatic treatment of Parkinson's disease, consists of stimulating periodically at a high frequency $(130 \mathrm{~Hz})$ the subthalamic nucleus in the basal ganglia [50-54], and leads to a remarkable reduction of motor symptoms and abnormal synchronization. Yet the mechanisms of action of DBS have remained elusive, and stimulation parameters are largely tuned heuristically and sometimes need to be readjusted following a subsequent emergence of neuropsychological symptoms [55-57]. Computational models speculated two possible mechanisms of action of DBS [58]: by increasing inhibitory currents and altering inhibitory firing pattern [59] or by depolarization blockade $[60,61]$. Here, we demonstrate that antiresonance in excitable networks could also serve as a hypothetical mechanism which, devoid of increased inhibition or excitation blockade, leaves the network highly responsive to stimuli and thus allows restoring cognitive processes. We will show that indeed, in the antiresonance regime, the system displays optimal information transmission capabilities, potentially joining clinical observations of DBS reportedly restoring motor and cognitive function in parkinsonian patients.

The paper is organized as follows. Section II introduces our reference neural network model, the electrically coupled FitzHugh-Nagumo network, and describes numerically the emergence of the noise-induced synchronization in this model. Section III is devoted to deciphering the dynamical mechanisms underpinning this synchronization, while Sec. IV unravels and analyzes the antiresonance phenomenon and the associated information transmission capabilities. We discuss the universality of these phenomena in Sec. V.

\section{MODEL AND NOISE-INDUCED SYNCHRONIZATION}

The electrically coupled network of FitzHugh-Nagumo neurons $[6,62]$ describes the dynamics of $n$ neurons through the equations:

$d v_{t}^{i}=\left[f\left(v_{t}^{i}\right)-w_{t}^{i}+\frac{J}{n} \sum_{j=1}^{n}\left(v_{t}^{j}-v_{t}^{i}\right)+I(t)\right] d t+\sigma d W_{t}^{i}$,

$d w_{t}^{i}=\varepsilon\left(b v_{t}^{i}-w_{t}^{i}\right) d t$,

where $i \in\{1, \ldots, n\}$ denotes the neuron index, $v^{i}$ the associated voltage, and $w^{i}$ the associated recovery variable. The function $f$ is a cubic nonlinearity modeling the excitability of the cells, classically considered as

$$
f(v)=v(1-v)(v-a),
$$

where $a>0$ controls the excitability, $J>0$ quantifies the coupling level, $I(t)$ is an input current, $\sigma$ is the level of noise, $\left(W_{t}^{i}\right)$ are independent Brownian motions, the timescale ratio $\varepsilon>0$ of the recovery variable compared to voltage is generally assumed small, and $b>0$ governs the coupling between voltage and recovery variable. Each neuron isolated, satisfying the FitzHugh-Nagumo equations, is thus classically an excitable system.

Coupling and noise have opposite effects on the collective dynamics: the former promotes coherence by pulling 
the voltage of each cell toward the average voltage the network generates, while noise reduces coherence by inducing random independent fluctuations of the voltage of each cell. Two regimes therefore arise for extremal values of coupling and noise (see Fig. 1 and Movie M.1 in Supplemental Material [63]).

Asynchrony.-For coupling sufficiently low relative to a fixed noise level, interactions become too weak to induce macroscopically organized dynamics, and neurons fire at random times as they cross the quasiseparatrix [Fig. 1(a), bottom]. As a result, asynchronous trajectories emerge, and the system reaches a stationary distribution. That distribution displays a bimodal shape, with a majority of neurons around the resting potential, and a macroscopic fraction in the spiking regime or in the course of firing [Fig. 1(b), bottom] [64].

Similarly, when noise is sufficiently large relative to a fixed coupling strength [66], neurons will fire asynchronously [Fig. 1(a), right]: intrinsic noisy fluctuations in those regimes overwhelm the dynamics and coupling terms, allowing neurons to spike independently of the state of other neurons. A broad stationary distribution ensues, covering both resting and excited parts of the phase plane, which has essentially a unimodal shape skewed toward the spiking region [Fig. 1(b), right].

Common to both asynchronous regimes, the distribution of neuron variables reaches a stationary state covering rest and excited regimes; the absence of rhythmic activity is visible in the low maximal amplitude of the Fourier transform of the average voltage or recovery variables [Fig. 1(c)].

Clamping.-For coupling sufficiently large (relative to a given noise level), or noise sufficiently small (relative to a coupling level), transitions to the excited regime are very rare, and the distribution of neurons remains clamped around the resting state [Fig. 1(a), top and left]. Heuristically, for coupling large, the interaction term becomes prominent compared to the intrinsic dynamics and dominates noisy fluctuations; this term forces each neuron to remain in the vicinity of the empirical average of the voltage, preventing noise from leading neurons into individual excursions [67]. For low noise, it is the rarity of transitions to the excited state that leaves the system passively "clamped" in the vicinity of the resting state.

Common to both clamped regimes, the empirical distribution of neurons concentrates at a stationary solution centered at the resting state [Fig. 1(b), left and top], and the low amplitude of the Fourier transform of the average voltage or recovery variables underlines the absence of rhythmic behavior [Fig. 1(c)].

While the above-described regimes can be readily understood heuristically, the transition between these two regimes is much more surprising, and is associated with noise-induced synchronization. (a) Trajectories

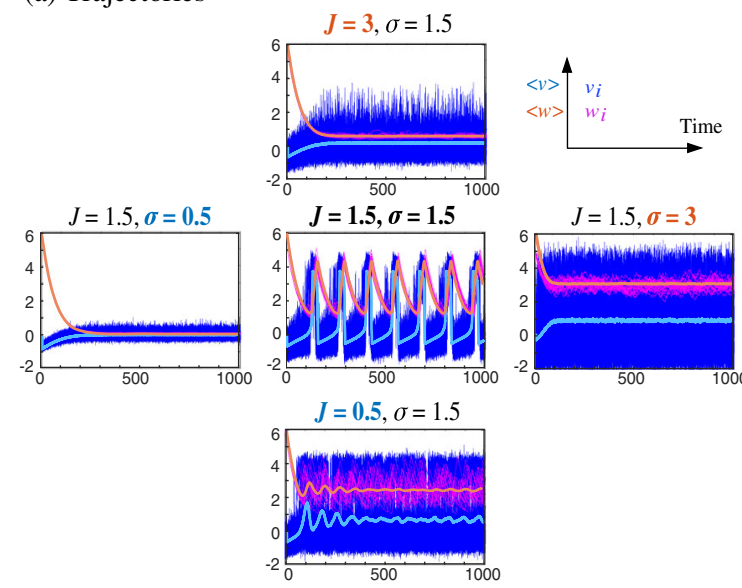

(b) Voltage distributions

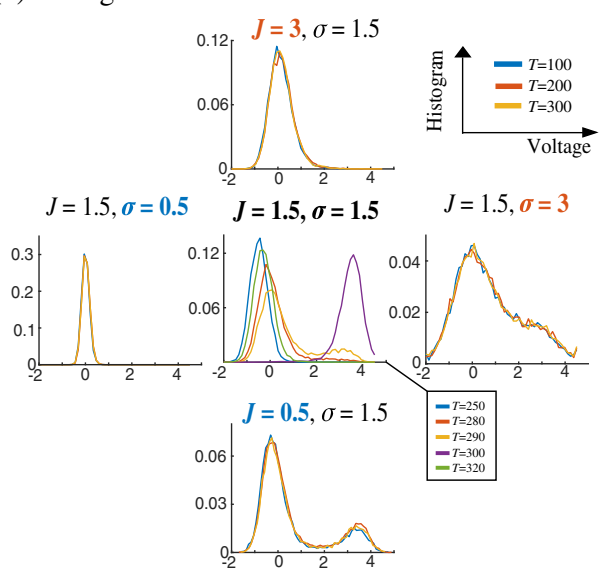

(c) Fourier analysis
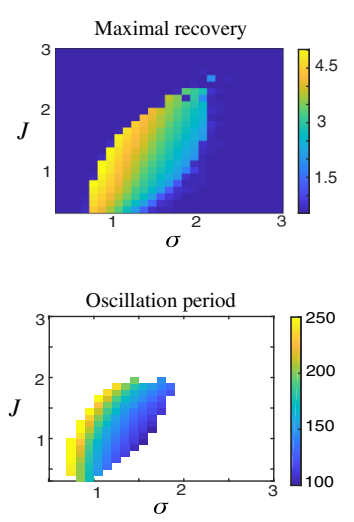

FIG. 1. Noise-induced synchronization in the FitzHugh-Nagumo model, with $n=4000$ neurons $[n=10000$ for histograms in (b)], $a=4, b=4, \varepsilon=0.01$, and $I=0$, for various values of the coupling strength $J$ and noise level $\sigma$. (a) Individual trajectories for 20 randomly chosen neurons in the network (dark blue, voltage; magenta, recovery variable), together with the average voltage (light blue) and recovery (pink). The system shows perfect collective synchrony at intermediate values of $J$ and $\sigma$ (here, $J=1.5$ and $\sigma=1.5$, center); small coupling (bottom, $J=0.5$ ) or large noise (right, $\sigma=3$ ) leads to asynchrony, high coupling (top, $J=3$ ) or low noise (left, $\sigma=0.5$ ) to clamping. (b) Distribution of the voltage variable in each regime and at different times: clamped regimes show a tight unimodal stationary distribution (no dependence in time), asynchronous regimes show either a broad (large noise, right) or a bimodal (low coupling, right) stationary distribution. In the noise-induced regime, the distribution is periodic; starting, e.g., around the resting state $(T=250$, blue $)$, it progressively widens and moves toward the excited regime, developing a bimodal shape as pioneers accumulate (yellow, $T=290$ ), until the whole distribution moves to the spiking region (purple, $T=300$ ) and comes back to rest (green, $T=320$ ). (c) Maximal amplitude (top) of the Fourier transform of the average recovery and associated period (bottom) highlighting a wide region of synchronized oscillations. 
Noise-induced synchronization.-For intermediate values of coupling and noise, the dynamics are no longer stationary: the trajectories display sharp, perfectly periodic, and synchronized macroscopic oscillations formed by all neurons firing synchronously within a small time interval [Fig. 1(a), center]. In this regime, high-amplitude oscillations of the distribution arise, as evidenced by the very peaked Fourier transform typical of periodic signals [Fig. 1(c)]. This nonstationarity is also visible in the distribution of the voltage at various times [Fig. 1(b), center].

The evolution of the distribution highlights the microscopic mechanisms supporting this phenomenon, and will be described in more detail in the following sections. Consider, for instance, an initial state centered in the vicinity of the resting state [blue curve in Fig. 1(b), center]. For noise sufficiently large and coupling sufficiently low, single neurons can overcome their attraction to the resting state, cross the quasiseparatrix, and reach the excited state (neurons performing these transitions will be called pioneers hereafter). These transitions do not lead to full spikes but partial deflections of the voltages that can lead to either a spike or a return to rest. Yet, as more neurons perform such transition, the voltage distribution progressively broadens and develops a peak in the excited region, gradually shifting the voltage of resting neurons closer to the quasiseparatrix, making transitions to pioneers more likely and thus the peak of the voltage distribution in the excited regime more prominent [yellow curve in Fig. 1(b), center] [68]. This progressive buildup is followed by a very rapid transition where all neurons eventually switch to the excited state, and, synchronized with the other neurons, perform a full collective spike (a macroscopic spike), associated with a very fast transition of the distribution to a unimodal one centered at the excited state. From that state, the distribution progressively returns to the vicinity of the resting state, where the process starts afresh.

The regime of noise-induced synchronization is not a singular transition between clamping and asynchrony: a relatively wide, eye-shaped region in the plane $(J, \sigma)$ corresponds to such oscillations [Fig. 1(c)], arising through a sharp transition from clamping (high-amplitude strongly periodic oscillations with very low frequency arise from clamping regimes) and smoothly transitioning to asynchrony. This suggests, similar to other observations in noise-induced oscillations [23,24], a transition via a Hopf bifurcation on the high-noise side and a homoclinic bifurcation on the low-noise side that will be shown to be a general observation in systems of coupled excitable elements in Sec. V.

\section{MICROSCOPIC PHENOMENA SUPPORTING NOISE-INDUCED SYNCHRONIZATION}

Noise-induced synchronization is thus associated with two remarkable phenomena: the buildup of a bimodal voltage distribution with spontaneous variations of the relative amplitude of the two peaks, as well as a sharp and sudden transition of all neurons to the excited regime where a macroscopic spike is emitted. We analyze here how the dynamics of each neuron contributes to the emergence of noise-induced synchronization.

In Sec. III A, we show that in the absence of noise, there exists a critical proportion of pioneers $\alpha=\alpha_{c}$ below which pioneers return to rest without firing a spike, and above which a chain reaction is triggered inducing a macroscopic spike. This leads us to conjecture that noise-induced oscillations arise when stochastic dynamics naturally lead the system to exceed this critical fraction. We thus study in Sec. III B the transition between resting and pioneer states, allowing us to account for the dynamics of the bimodal distribution and to delineate regimes where the network spontaneously reaches the critical fraction of pioneers needed to synchronize (thus, enters the noise-induced oscillations regime) or remains below that threshold (leading to stationary solutions).

\section{A. Nonlinear dynamics of macroscopic spikes}

Macroscopic spikes stand out as sudden, sharp, and dramatic events affecting all neurons, and arising as the fraction of neurons in the excited state progressively increases. We show here that this sudden switch can be associated with a nonlinear change of the stability of the resting state as the number of pioneers increases, independently of the stochastic fluctuations.

To this purpose, we investigate the behavior of a set of $n$ neurons satisfying Eq. (1) in the absence of noise as the initial fraction of neurons in the pioneer state $\alpha$ is varied. For $\alpha$ sufficiently small, we indeed observed no macroscopic spike generated (Fig. 2): the maximal value of the average voltage remains bounded below the spike level [Fig. 2(a)], and trajectories of the system show a direct return of all neurons to rest [Fig. 2(b)]. However, a sudden and sharp transition occurs at a critical fraction of pioneers $\alpha_{c}$ : the presence of a sufficient number of pioneers produces a strong attraction toward the excited state, leading to a chain reaction during which all remaining resting neurons transition to pioneers, followed by a macroscopic spike. For these initial levels of pioneers, the maximal value of the average voltage jumps to the spiking level [Fig 2(a)] and the average trajectory in the phase plane [Fig. 2(b)] displays a spike and return to rest.

To determine analytically $\alpha_{c}$ and quantify precisely its dependence upon $J$, we consider the nonlinear evolution of the average voltage of resting $\left(v_{1}\right)$ and pioneer $\left(v_{2}\right)$ neurons assuming that the transition occurs in a timescale faster than the recovery variable (i.e., recovery variable considered fixed equal to some value $w_{0}$ ), a relevant approximation here owing to the slow evolution of the recovery variable during the transition [69]. For a given proportion of pioneers $\alpha$, the 
(a) Existence of a critical fraction of pioneers
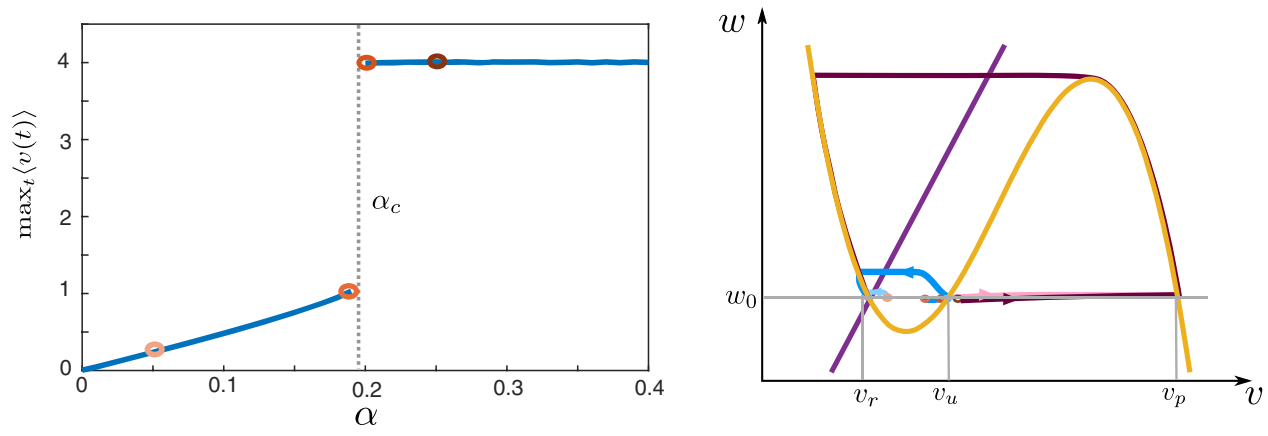

(b) Bifurcation diagram
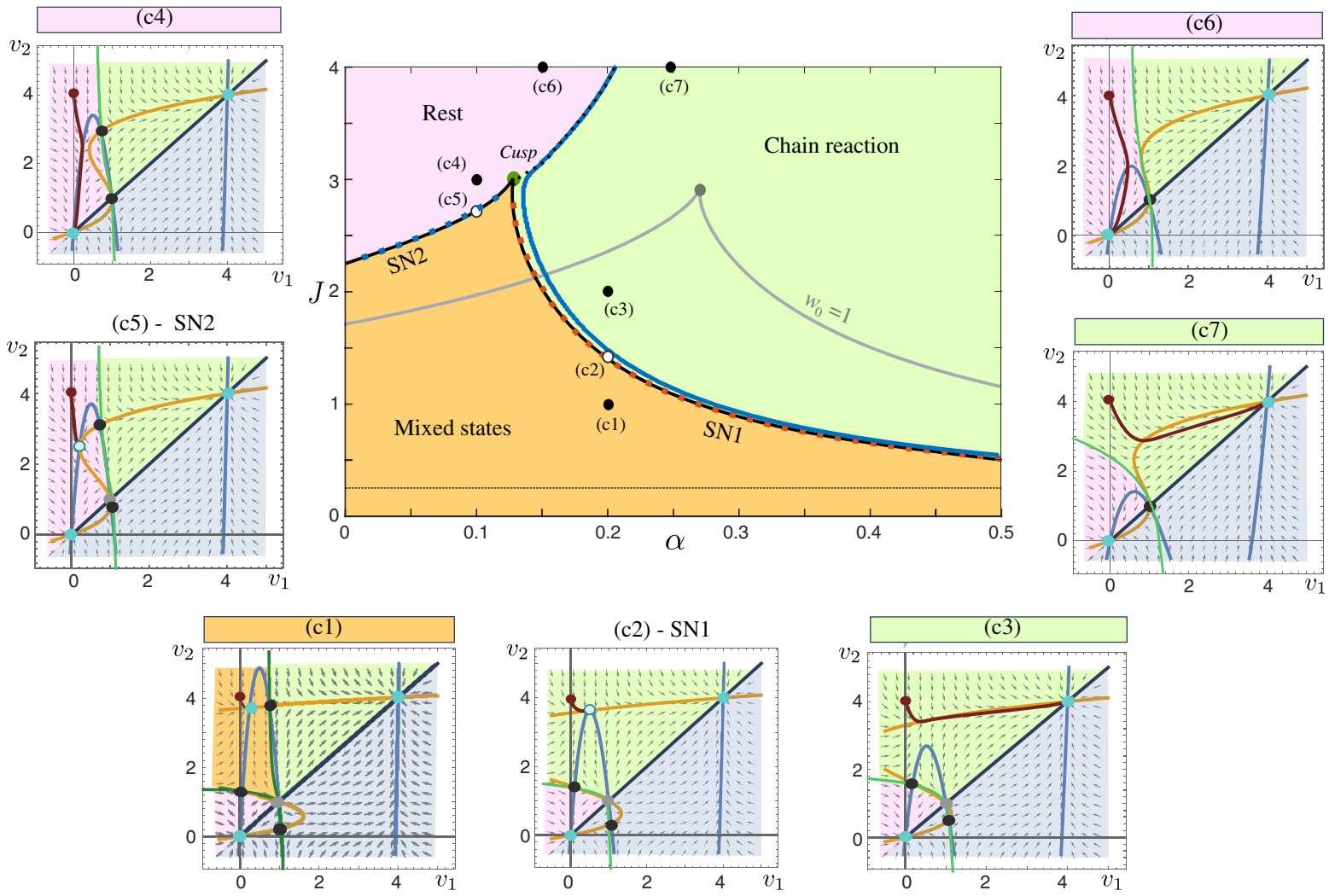

FIG. 2. Chain reaction. (a) Left: Maximal value of the average voltage for network [Eq. (1)] in the absence of noise $(\sigma=0)$ as a function of the initial proportion of pioneers $\alpha(n=1000, a=4, \varepsilon=0.01, b=4, J=1.5, I=0)$ shows a sudden discontinuity at a critical value $\alpha_{c}$, where (right) trajectories in the phase plane (yellow, $v$ nullcline; purple, $w$ nullcline) switch from rapid returns to rest for $\alpha<\alpha_{c}$ (light blue, $\alpha=0.05$; blue, $\alpha=0.19$; lighter two shades of red circles in the right-hand diagram) to long collective spiking excursions for $\alpha>\alpha_{c}$ (pink, $\alpha=0.21$; red, $\alpha=0.25$; darker two shades of red circles in the right-hand panel). Initial condition (averaged) is depicted by a colored circle. Gray line, $w_{0}$, depends on the level of noise; the values $v_{r}, v_{u}$, and $v_{p}$ are intersections of the line $w=w_{0}$ and the cubic nullcline. (B) Critical fraction of pioneers associated with the chain reaction as a function of coupling $J$ in the two-dimensional system [Eq. (1)] (blue curve) or in the simplified equation (2) (dashed blue, transition from rest to mixed equilibria; dashed red, transition from mixed equilibria to pioneers; dotted black, transition from rest to pioneer), together with the bifurcations of the simplified one-dimensional model (2) with $w_{0}=0$ (black) or $w_{0}=1$ (gray), showing two saddle-node bifurcations curves (SN1 and SN2) joined at a cusp bifurcation. (c1)-(c5) Typical phase portraits of the simplified system [Eq. (2)]. Dark blue, identity line (irrelevant phase space grayed); yellow and blue curves, $v_{1}$ and $v_{2}$ nullclines; cyan circles, stable fixed points; gray circles, unstable fixed points; black circles, saddles. Green lines represent the stable manifold of the saddles and partition the phase space into the attraction basin of stable fixed points [green, chain reaction; orange, mixed equilibrium; pink, rest, as in (c)]. Arrows represent the direction of the flow. The dark red curve represents the trajectory starting from $v_{1}=v_{r}$ and $v_{2}=v_{p}$. (c1) $\alpha=0.2$ and $J=1$, (c2) $\alpha=0.2$ and $J=1.4$ near SN1, (c3) $\alpha=0.2$ and $J=2$, (c4) $\alpha=0.1$ and $J=3$, (c5) $\alpha=0.1$ and $J=2.73$ near SN2, (c6) $J=4$ and $\alpha=0.15$, (c7) $J=4$ and $\alpha=0.25$. 
evolution of $v_{1}$ and $v_{2}$ is thus approximated by the twodimensional ordinary differential equation (ODE):

$$
\begin{aligned}
& v_{1}^{\prime}=f\left(v_{1}\right)-w_{0}+J \alpha\left(v_{2}-v_{1}\right), \quad v_{1}(0)=v_{r}, \\
& v_{2}^{\prime}=f\left(v_{2}\right)-w_{0}-J(1-\alpha)\left(v_{2}-v_{1}\right), \quad v_{2}(0)=v_{p} .
\end{aligned}
$$

where $w_{0}$ corresponds to a recovery variable value such that the dynamics of a single particle (uncoupled system).

$$
v^{\prime}=f(v)-w_{0},
$$

display three equilibria: a resting state $v_{r}$ on the leftmost branch of the cubic function $f$, a pioneer state $v_{p}$ on the rightmost branch [values appearing as initial conditions in Eq. (2)], and an unstable fixed point $v_{u}$ on the middle branch splitting the attraction basins of the two equilibria [70]. We are interested in solutions of these equations with $v_{1} \leq v_{2}$.

The dynamical system (2) features at least three fixed points with identical voltage, $v_{1}=v_{2} \in\left\{v_{r}, v_{u}, v_{p}\right\}$ [Fig. 2(b)]. These fixed points have the same stability as the associated voltage in the one-dimensional uncoupled FitzHugh-Nagumo system (3). Indeed, the Jacobian matrix of the system (2) at an arbitrary point $\left(v_{1}^{*}, v_{2}^{*}\right)$ reads:

$$
\left(\begin{array}{cc}
f^{\prime}\left(v_{1}^{*}\right)-J \alpha & J \alpha \\
J(1-\alpha) & f^{\prime}\left(v_{2}^{*}\right)-J(1-\alpha)
\end{array}\right) .
$$

For $v_{1}^{*}=v_{2}^{*}=v_{r}$ or $v_{p}$ [the two stable fixed points of Eq. (3)], the trace of the Jacobian matrix is strictly negative and its determinant $f^{\prime}\left(v^{*}\right)^{2}-J f^{\prime}\left(v^{*}\right)$ is strictly positive [since $f^{\prime}\left(v^{*}\right)<0$ for a stable fixed point], implying stability of the fixed points $\left(v_{r}, v_{r}\right)$ and $\left(v_{p}, v_{p}\right)$ for the two-dimensional system. If $v_{1}^{*}=v_{2}^{*}=v_{u}$, the unstable fixed point of Eq. (3), the trace reads $2 f^{\prime}\left(v_{u}\right)-J$ and the determinant $f^{\prime}\left(v^{*}\right)\left[f^{\prime}\left(v^{*}\right)-J\right]$. The determinant is nonnegative when $f^{\prime}\left(v^{*}\right)>J$, in which case the trace is positive. The fixed point $\left(v_{u}, v_{u}\right)$ is thus always unstable for system (2): it is a saddle when $f^{\prime}\left(v^{*}\right)<J$ and an unstable node for $f^{\prime}\left(v^{*}\right)>J$.

The equilibria with identical voltage correspond to a synchronization of the network: $\left(v_{r}, v_{r}\right)$ corresponds to all pioneers returning to rest, and $\left(v_{p}, v_{p}\right)$ to all resting neurons reaching the excited state. In addition to these attractors, mixed equilibria (i.e., with $v_{1}<v_{2}$ ) may also exist, when the system is able to support a mixture of a fraction $\alpha$ of pioneers and $(1-\alpha)$ of resting neurons (e.g., in the asynchrony regime). A chain reaction occurs if, starting from the initial condition $\left[v_{1}(0)=v_{r}\right.$, $\left.v_{2}(0)=v_{p}\right]$, the system reaches the fixed point $\left(v_{p}, v_{p}\right)$, or in other words, $\left(v_{r}, v_{p}\right)$ belongs to the attraction basin of $\left(v_{p}, v_{p}\right)$. We thus analyzed the geometry of the phase plane of system (2), and found that, as parameters are varied, the initial condition $\left(v_{r}, v_{p}\right)$ could suddenly switch attraction basins, either associated with smooth changes in the shape of these basins or organized by the stable manifold of a saddle mixed equilibrium [Fig. 2(c)].

To characterize these transitions, we first computed the two-parameter bifurcation diagram of Eq. (2) as a function of the coupling strength $J$ and the proportion of pioneers $\alpha$. This diagram displays two branches of saddle-node bifurcations (black curves) merging at a cusp bifurcation. These saddle-node curves delineate the region where mixed equilibria exist. We found that these bifurcations coincide exactly with transitions in the eventual state of the system starting from the initial condition $\left(v_{r}, v_{p}\right)$ (dashed blue and red curves, obtained by extensive simulations of the system). Moreover, we recover precisely the value $\alpha_{c}$ corresponding to the transition found for the full system (1) at $J=1.5$ and with $\sigma=0$ [Fig. 2(a)]. More generally, when $J$ is varied, we found an excellent agreement between the critical fraction $\alpha_{c}$ and the evaluations obtained with the simplified system (2) (blue curve in Fig. 2).

The coincidence between saddle-node bifurcations and switches in the attractor to which solutions of system (2) converge can be inferred analyzing the geometry of vector field of Eq. (2). Attraction basins in this twodimensional system are governed by the stable and unstable manifolds of the saddle fixed points, depicted in green in Figs. 2(c1)-2(c7). We observe that whether or not the initial condition belongs to the attraction basin of rest or pioneer strongly depends on the presence of mixed saddles (rather than the specific shape of those manifolds), accounting for the perfect agreement between saddle-node bifurcations and switches in the fixed point toward which the system converges. Mixed equilibria do not exist for $J$ larger than the value associated with the cusp. For such parameters, $\left(v_{u}, v_{u}\right)$ is a saddle, and its stable manifold splits the phase space into the attraction basins of $\left(v_{r}, v_{r}\right)$ and $\left(v_{p}, v_{p}\right)$. Smooth changes in the shape of that manifold lead to a switch between resting and pioneer, and the associated value of $\alpha$ associated with the transition is represented as a dotted line in Fig. 2 and closely agrees with the chain reaction separatrix (blue curve) computed for the full system.

Clamping at high coupling strength may be associated with the incapacity of the system to support a mixture of pioneer and resting neurons for $J$ larger than the value associated with a cusp (the full system returning to rest either directly of after one spike depending on the initial $\alpha$ ). In sharp contrast, coupling values $J$ small enough [below the dashed line in Fig. 2(c)] allow the existence of mixed equilibria for any initial proportion of pioneers $\alpha$ : coupling is not sufficient to promote a collectively coherent behavior, leading to asynchronous behaviors.

The bifurcations and transitions found depend on the value of $w_{0}$, which, in the original model with noise (1), depends both on $\sigma$ and $J$. To appreciate how $w_{0}$ alters the transitions found in the system, we computed the twoparameter bifurcation diagram of system (2) as a function 
of $\alpha$ and $J$ for a distinct value of $w_{0}$ [Fig. 2(c), gray curve, $\left.w_{0}=1\right]$, and found a qualitatively similar bifurcation diagram as originally obtained for $w_{0}=0$, but essentially shifted to larger values of $\alpha$. Heuristically, a larger $w_{0}$ would correspond to larger noise (or weaker coupling), and the system would necessitate a larger fraction of pioneers to display the chain reaction. The absence of qualitative dependence in $w_{0}$ was confirmed in Fig. 13 representing the two-parameter bifurcation diagram of system (2) as a function of $\alpha$ and $w_{0}$ for distinct values of $J$. We found monotonically increasing bifurcation lines and no codimension-two bifurcation, showing that the qualitative features outlined above persist for various values of $w_{0}$.

We thus conclude that a chain reaction may arise when the population is composed of a sufficiently large fraction of pioneers. We now relax our assumption of considering fixed values of $\alpha$, and turn our attention to the stochastic transitions between resting and pioneer that govern the evolution of $\alpha$.

\section{B. Reaching the critical proportion of pioneers}

The question that arises is thus whether the critical fraction of pioneers $\alpha_{c}$ is reached spontaneously by the stochastic system. In the presence of noise, neurons at rest may indeed transition to pioneer, and reciprocally Eq. (1); the fraction of pioneers will thus vary in time according to the rates of transitions. To describe this evolution, we now quantify the rates at which resting neurons transition to pioneers and reciprocally. Figure 12 in Supplemenal Material represents the evolution of the fraction of pioneers in various situations [63].

\section{Stochastic transitions between pioneer and resting states}

Characterizing the rate of transition of a stochastic particle in a multiwell potential is a classical and widely studied question in the domain of stochastic analysis [71,72]. Most results are derived in the small noise limit and for Hamiltonian systems. The problem of noise-induced synchronization we are studying here challenges classical theory in many ways. In particular, noise-induced oscillations arise for nonvanishing noise, the dynamics are not Hamiltonian, noise modifies the dynamics (potential and equilibria in a Hamiltonian analogy), and the transitions are collective; i.e., transition rates depend on the positions of all other particles through the coupling term. In Sec. A 1 of Supplemental Material, we discuss in more detail these questions, highlight the difficulty to define a double-well potential for the system and how that putative potential depends on noise and on the distribution of neuron voltages (chiefly through $\alpha$ ), and how theoretical estimates may deviate from the effective rates of transitions [63].

To numerically evaluate the transition rates from rest to pioneer and reciprocally, we again reduced the system to a one-dimensional equation on the voltage only, assuming a fixed value of the recovery variable $w=w_{0}$ during the transition phase. Under this hypothesis, and between two transitions, each neuron satisfies the equation:

$d v_{t}=\left\{f\left(v_{t}\right)-w_{0}+J\left[(1-\alpha) v_{r}+\alpha v_{p}-v_{t}\right]\right\} d t+\sigma d W_{t}$.

where $\alpha$ is the current fraction of pioneers (fixed between two consecutive transitions) and $v_{p}$ and $v_{r}$ are the pioneer and resting voltage, approximated as the largest and smallest solution of

$$
f(v)=w_{0} .
$$

The first step of this program thus consists in evaluating $w_{0}$ numerically as a function of those parameters. To this end, we simulated the full system Eq. (1) and computed the median value of the recovery variable at transitions from rest to pioneer [see Fig. 3(b)] [73]. Using this estimate, we next systematically evaluated, for various pairs of $(J, \sigma)$, the distribution of transition times for a particle satisfying Eq. (4) for multiple (typically, 15 000) realizations of the process with independent noise and independent random initial condition within the pioneer or resting regime of the uncoupled system [74].

A particle is considered to switch attractors if, starting on one side of the quasiseparatrix (embodied by the unstable fixed point $v_{u}$ ), it ends up dwelling on the other side. To avoid considering transient passages of a particle through the quasiseparatrix not corresponding to actual transitions, we used a confidence interval to determine transition times: a pioneer [rest] neuron was considered to have switched to rest [pioneer] if its voltage exceeds $\gamma v_{r}+(1-\gamma) v_{u}$ [goes below $\gamma v_{p}+(1-\gamma) v_{u}$ ] with $\gamma=0.1$ [75]. We observed that the transition times are exponentially distributed with a rate that depends upon the proportion of pioneers [see Fig. 3(a)]. We confirmed that the data are indeed statistically consistent with an exponential distribution fitting a rate using the maximum likelihood estimator and a Kolmogorov-Smirnov test. For $\alpha=0.2$ [Fig. 3(a)], we found a rate of transition from pioneer to rest (rest to pioneer) equal to $\lambda=0.20(\lambda=0.17)$, with a goodness of fit (Kolmogorov-Smirnov) 0.013 (0.0153) and a $p$ value $p<0.015$ ( $p=0.017)$ (see Ref. [76] for details on the statistical test and code), confirming that the data are perfectly consistent with an exponential.

Finding an exponential distribution opens the way to an important simplification of the system. Indeed, because exponential transitions are memoryless, the evolution of the proportion of pioneers can now be modeled as a continuous-time finite-state Markov process. In detail, assuming that the distinct neurons have independent and identically distributed transition times (the property that finite subsets of neurons are independent and identically distributed in this system was demonstrated mathematically in Ref. [65], 
(a) Exponential distribution of transition times

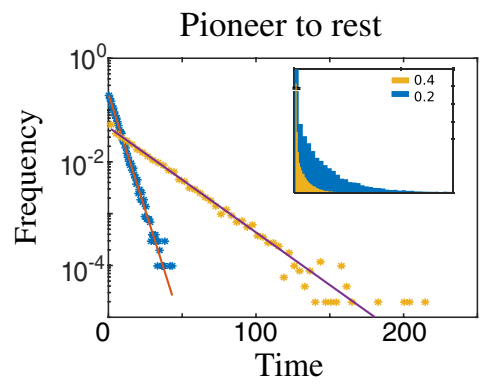

(c) Transition rates
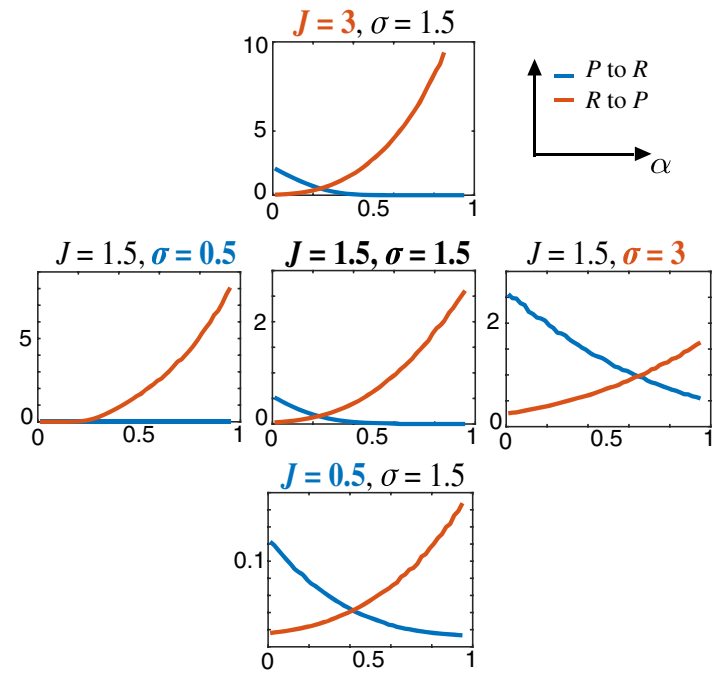

(b) Variation of $w_{0}$

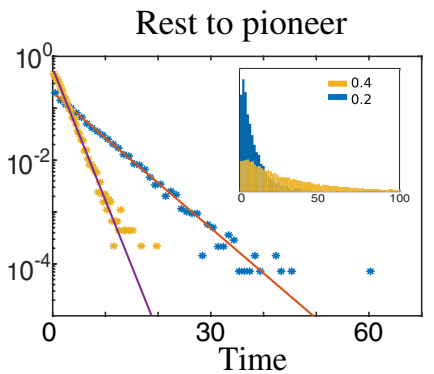

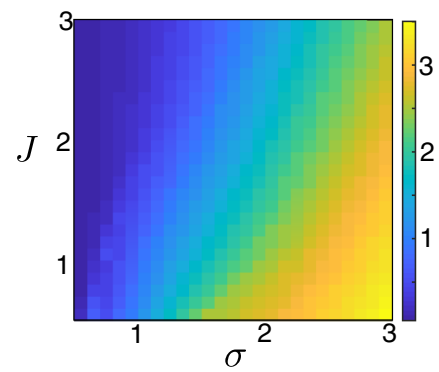

(d) Flow of the fraction of pioneers

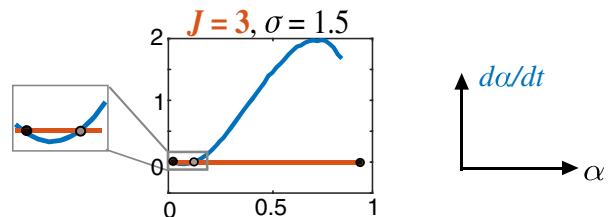

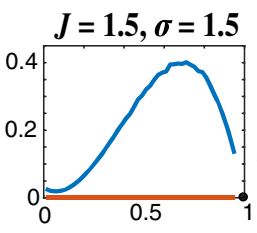
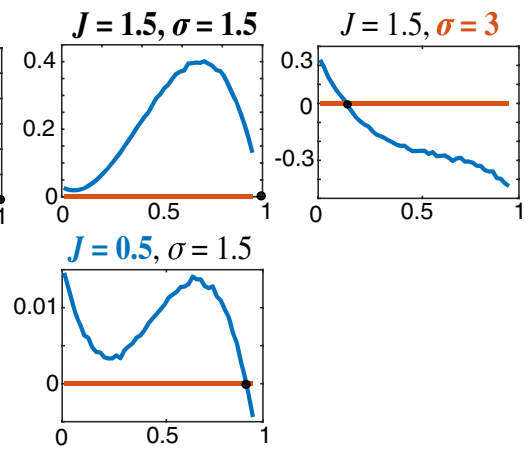

FIG. 3. Stochastic transitions between resting and pioneer states. (a) Histogram of the transition time from pioneer to rest (left) and rest to pioneer (right), in semilogarithmic scale (inset: linear scale) for $\alpha=0.2$ (blue) or $\alpha=0.4$ (yellow). The corresponding maximum likelihood fit with an exponential distribution shows an excellent match (solid lines, red, $\alpha=0.2$; purple, $\alpha=0.4$ ). Parameters as in Fig. 1 with $J=1.5, \sigma=1.5$, and $\alpha=0.2$; a sample 15000 independent simulations of Eq. (4) was used. (b) Variation of $w_{0}$ as a function of $\sigma$ and $J$, computed as the median value of recovery variable at the transition for the original system with $n=4000$ neurons. (c) Transition rates obtained from the maximum likelihood estimator with the exponential distribution as a function of $\alpha$ [blue, pioneer to rest; red, rest to pioneer; 15000 simulations of Eq. (4) for the five situations considered in Fig. 1(a)]. (d) Flow of the fraction of pioneers [right-hand side of Eq. (5), blue curve versus 0 in red].

a property referred to as the propagation of chaos property in mathematics), the number of pioneers $\left(P_{t}\right)_{t \geq 0}$ forms a birth-and-death Markov process on the finite-state space $\{0, \ldots, n\}$ with transitions

$x \rightarrow x+1$ with rate $(n-x) K_{R P}\left(\frac{x}{n}\right), \quad$ for any $x<n$,

$x \rightarrow x-1$ with rate $x K_{P R}\left(\frac{x}{n}\right), \quad$ for any $x>0$,

where $K_{R P}$ and $K_{P R}$ are the transition rates from rest to pioneer and reciprocally. Therefore, for $n$ large, using the Kolmogorov equation for that Markov chain, we find that the proportion of pioneers at time $t, \alpha(t)=P_{t} / n$, satisfies the ordinary differential equation:

$$
\frac{d \alpha}{d t}=(1-\alpha) K_{R P}(\alpha)-\alpha K_{P R}(\alpha) .
$$

The fixed points of that one-dimensional equation, representing the steady-state proportions of pioneers, are given by the implicit equation

$$
\alpha^{\star}=\frac{K_{R P}\left(\alpha^{\star}\right)}{K_{R P}\left(\alpha^{\star}\right)+K_{P R}\left(\alpha^{\star}\right)},
$$

and these are stable equilibria when

$$
\left(1-\alpha^{\star}\right) K_{R P}^{\prime}\left(\alpha^{\star}\right)-\alpha^{\star} K_{P R}^{\prime}\left(\alpha^{\star}\right)-\left[K_{R P}\left(\alpha^{\star}\right)+K_{P R}\left(\alpha^{\star}\right)\right]<0 .
$$

To determine equilibrium proportions of pioneers and their stability as a function of the parameters, we systematically evaluated the distribution of transition times for various values of $\alpha$ and fitted an exponential distribution using the maximum likelihood estimator (15000 samples for each condition). We observed, as expected, that the rate 
of transition from rest to pioneer increases with $\alpha$ while the rate of the reciprocal transition decreases [see Fig. 3(c)]. Indeed, the larger $\alpha$, the faster resting neurons reach the pioneer state, while pioneer states are stabilized for $\alpha$ large and transitions to rest rarer. From these transition curves, we computed the flow of $\alpha$ [Eq. (5)] as a function of coupling $J$, noise $\sigma$, and proportion of pioneers $\alpha$. We observe clear transitions in the shape of the flow, associated with the transitions from clamping to synchrony and then asynchrony, as visible in Fig. 3(d) for the values of $J$ and $\sigma$ highlighted in Fig. 1 [see also Fig. 4(b) for a more exhaustive representation of the flow, corrected by the spontaneous spike rate of pioneers introduced in Sec. III B 2].

(a) Inverse of spike duration

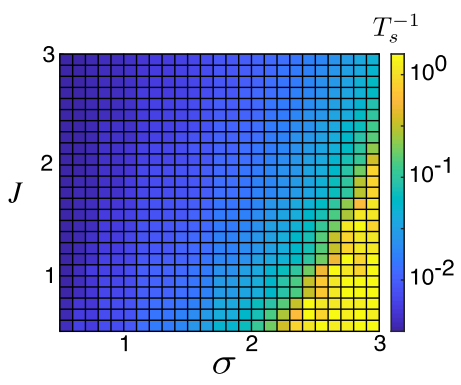

(c) Flow of the fraction of pioneers

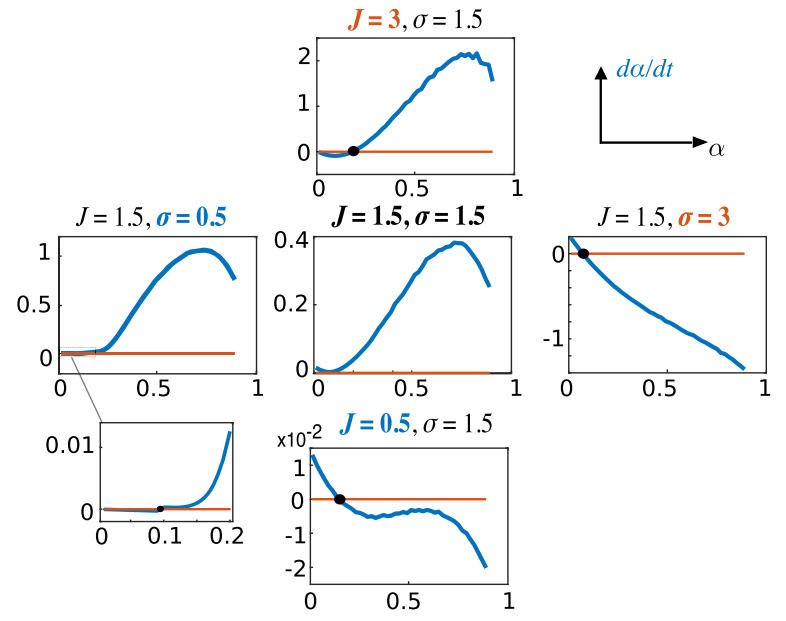

Although our numerical estimations neglect the transitions from pioneers to rest subsequent to a spike (introduced in the next section), it already accounts for three of the five regimes observed in Fig. 1.

(i) In the synchronized regime [central regime in Fig. 1(a)], the flow is uniformly positive, indicating that $\alpha$ is strictly increasing regardless of the initial condition. Therefore, neurons accumulate rapidly within the pioneer region, reach the critical proportion $\alpha_{c}$, undergo the chain reaction through a macroscopic spike, and return to $\alpha=0$ where the process starts afresh. Because the typical time for one given transition is significantly smaller than the time of a spike (see Sec. III B 2), the model accurately

(b) Flow of the fraction of pioneers

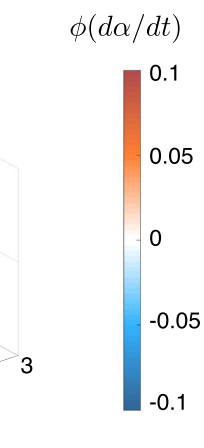

(d) Agreement with the stochastic network model
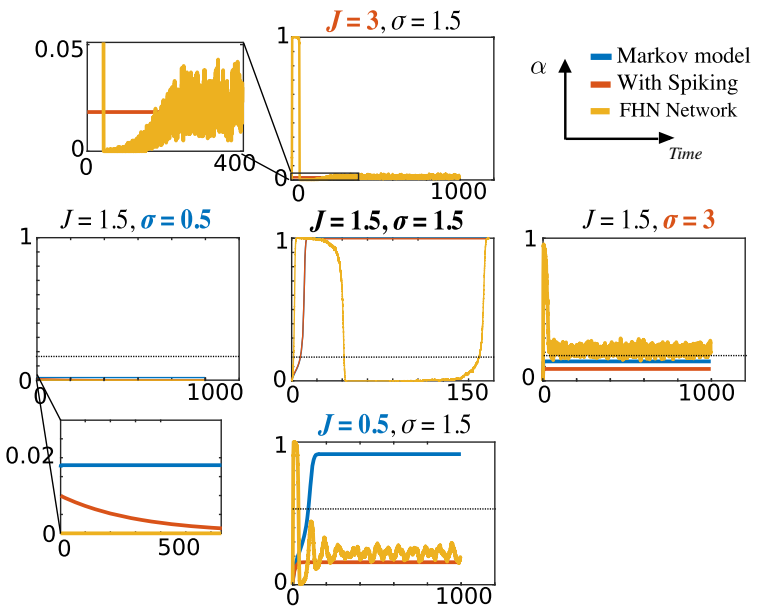

FIG. 4. Stochastic and spiking transitions. Combining stochastic transitions and spiking recovers qualitatively and quantitatively the phenomenology of the stochastic FitzHugh-Nagumo network of Fig. 1. (a) Numerical evaluation of the inverse spike duration [Eq. (6)] as a function of $\sigma$ and $J$. (b) Flow of the fraction of pioneers [right-hand side of Eq. (7)] as a function of $\sigma, J$, and $\alpha$ (fourdimensional representation using PCOLOR3 MATLAB routine [77]). The system recovers the clamping fixed point with 0 pioneers in the low-noise or high-coupling regimes, nontrivial fixed points for large-noise or low-coupling regimes, and a uniformly positive flow (chain reaction and noise-induced synchronization) for intermediate values (red spherelike surface). For legibility, the flow was smoothed and thresholded through the function $\phi: x \mapsto[1+\tanh (4 x)] / 2$. (c) Flow of the fraction of pioneer $\alpha$ in the five situations considered in Fig. 1(a), recovers the appropriate dynamics in all cases qualitatively. (d) Number of pioneers in (red) the simplified model (7) taking into spiking, the simplified model neglecting spiking (blue), and the stochastic network (yellow). Good agreement is found with the simplified model when taking into account spiking, deviating significantly from the model neglecting spikes especially at low coupling or low noise. When it exists, the critical fraction of pioneers $\alpha_{c}$ associated with the chain reaction is depicted as a black dashed line. 
accounts for the observations on the full system. Moreover, we note that the time for the proportion of pioneers to reach $\alpha_{c}$ is deterministic [it is the crossing time of $\alpha_{c}$ of the solution of Eq. (5) starting from $\alpha=0$ ], accounting for the regularity of the periodic behavior observed in Fig. 1.

(ii) In the clamped regime for large $J$ [top, Fig. 3(d)], we observe that the fraction of pioneers shows bistable dynamics, with a stable fixed point at $\alpha=0$ (clamped state), a stable fixed point at $\alpha=1$ (chain reaction regime), and a third unstable fixed point separating the attraction basins of both equilibria near the chain reaction threshold [for large $J, w_{0}$ is close to 0 and the chain reaction threshold is slightly below 0.2, see Fig. 2(d)].

Depending on the initial $\alpha$, either all neurons return to rest, or all neurons reach the pioneer state, fire a macroscopic spike, and return to the clamped regime near equilibrium. The computed curves seem to indicate that this transition occurs through a saddle-node bifurcation in the rate equation (2), as the quadratic behavior of the flow for small $\alpha$ progressively shifts up and becomes tangent to the 0 line. As the system approaches the transition, the time taken by the system to trigger a spike decays to zero, accounting for the notable slowing down of the oscillations near this transition (see Fig. 1).

(iii) Eventually, for large $\sigma$, the transition rate from pioneer to rest and back again shows a less sensitive dependence in $\alpha$ : noise becomes sufficient to induce transitions in both directions for any value of $\alpha$ and starts dominating the interaction terms. The fraction of pioneers $\alpha$ thus rapidly converges to the unique stable fixed point of the associated system, close to 0.2 here. At this value of $\alpha$, the number of resting neurons transitioning to pioneer is balanced by the number of pioneers returning to rest, and a stationary asynchronous firing regime ensues.

In all these three cases, the rates of transitions are relatively large compared to the duration of a spike, and therefore the accumulation of pioneers and transient variations of $\alpha$ occurs prior to any neuron firing a spike. This is not the case of low-noise clamping or low-coupling asynchronous regimes. In the low-noise regime, both rates of transitions are very low. In the low-coupling case, rates of transitions are no more low, but the transition from rest to pioneers is compensated by the reciprocal transition arising at a similar rate. In both cases, the very slow evolution of the fraction of pioneers ensuing allows neurons that have transitioned early to pioneers to spike and return to rest before accumulation of neurons in the pioneer state. This phenomenon shall be crucial when the typical time for reaching critical value $\alpha_{c}$ is larger than the typical spike duration (or when it is infinite, i.e., when $\alpha_{c}$ is never reached).

\section{Modeling returns to rest after spiking}

To account for this phenomenon, we added a corrective term to Eq. (5) considering the rate at which pioneers return to rest after spiking. To this end, we computed the typical time for a pioneer to fire a spike. The spike is composed of three main phases: an upstroke where the voltage climbs up the rightmost branch of the stable part of the cubic nullcline from $w=w_{0}$ up to a value $w_{1}$ where typical trajectories leave that branch (much like $w_{0}$, the value of $w_{1}$ depends on noise and coupling), a rapid jump to the leftmost branch of the cubic at $w=w_{1}$, and then a downstroke where the trajectory decays along the right branch of the cubic down to $w=w_{0}$. Neglecting the rapid switching time between the upstroke and downstroke phase, we can express analytically the spike duration as

$$
T_{s}=\frac{1}{\varepsilon}\left(\int_{w_{0}}^{w_{1}} \frac{d w}{b v_{p}(w)-w}+\int_{w_{1}}^{w_{0}} \frac{d w}{b v_{r}(w)-w}\right)
$$

where $v_{p}$ and $v_{r}$, as defined above, are the right and left solutions of the cubic polynomial equation,

$$
f(v)-w+I=0 .
$$

The above formula highlights the fact that the spike duration is of order $\varepsilon^{-1}$, much longer compared to the stochastic fluctuations, but not necessarily longer than the time needed to accumulate neurons in the pioneer state. We thus used the above formula and the classical analytical expressions of $v_{r}$ and $v_{p}$ (we chose the trigonometric form for $v_{r}$ and $v_{p}$ due to François Viète) and a numerical evaluation of $w_{1}$ (similar methodology as used for $w_{0}$ ) to compute the spiking time.

We used this estimate of the typical spike duration, and the fact that $n$ is considered large, to model the fraction of pioneers returning to rest after a spike as a ceaseless leak of the density of pioneers to rest at a rate $T_{s}^{-1}$ :

$$
\frac{d \alpha}{d t}=(1-\alpha) K_{R P}(\alpha)-\alpha\left[K_{P R}(\alpha)+T_{s}^{-1}\right] .
$$

We confirmed that this correction is essentially negligible in the case of noise-induced oscillations, large-noise asynchrony, or large-coupling clamping, but has important qualitative implications in the low-noise or small-coupling regimes, as visible in Fig. 4. Indeed, we observe that for small noise, very small values of $\alpha$ are stabilized, owing to the rarity of transitions at low $\sigma$. More generally, in the clamping regime the system stabilizes to a fraction of pioneers $\alpha$ at which transitions from rest to the excited state are compensated by stochastic and firing returns: small perturbations of this equilibrium toward smaller $\alpha$ will progressively be compensated by noise, and a slight deviation toward a higher $\alpha$ below the chain reaction will be damped as neurons fire a spike and return to rest. In this 
regime, however, the dynamics are relatively sensitive, as the flow converges, when $\sigma \rightarrow 0$, to a discontinuous flow (with a discontinuity at the critical $\alpha=\alpha_{c}$ associated with the chain reaction). This sharpness is visible in our simulations (Fig. 4).

When coupling is small, the corrected system taking into account the return of pioneers to rest through a spike also recovers the observed behavior of the emergence of a stable fixed point. In this regime, collective behaviors are less likely to arise as each cell is mostly driven by its own noise and excitable dynamics, essentially dominating the coupling term. Because of low coupling, while rates of transition remain of the same order of magnitude, the absence of a strong influence of network dynamics implies that the rates essentially compensate. We indeed observe that transition times are of the same order of magnitude of the duration of a spike [notice the maximal rate of transition on the order of $15 \times 10^{-3}$ in Fig. 3(c)]. Therefore, adding the return to rest term associated with spikes has a highly nontrivial impact, and yields the emergence of a stable fixed point for $\alpha$ below the critical value, indicating the emergence of a stationary probability to be in the pioneer state. In other words, because neurons are asynchronous, the fraction of neurons in the pioneer or rest regime is the stationary mass of the distribution of a single neuron within those sets.

We confirmed that the model precisely accounts for the evolution of the number of pioneers as a function of time, starting from $\alpha=0$. We depict in Fig. 4(d) the fraction of pioneers computed in the stochastic FitzHugh-Nagumo network Eq. (1) together with the simple one-dimensional differential equation (7), and observe that our model, despite its simplicity, finely accounts quantitatively for the number of pioneers. For instance, in the noise-induced oscillations regime, the model reflects the slow transitions arising in the system until the system reaches the critical proportion of pioneers $\alpha_{c}$, at which time a very sharp increase of $\alpha$ arises.

In Fig. 4(b), we provide an extensive view of the dynamics of $\alpha$ as a function of $J$ and $\sigma$ in a four-dimensional representation [color represents the righthand side of Eq. (7)]. This representation recovers the eye-shaped noise-induced synchronization regime, corresponding to the parameter values associated with a uniformly positive value for intermediate values of noise and coupling.

\section{Conclusion: A subtle interplay of noise, connectivity, and excitability}

This analysis of the trajectories provides a novel dynamical view of synchronization of stochastic particles. By finely dissecting the mechanisms of noise-induced synchronization in the FitzHugh-Nagumo network, we identified three distinct regimes of dynamics: synchrony for intermediate noise and coupling, flanked by asynchrony (large noise or low coupling), and clamping (low noise or high coupling). The analysis of the dynamics of single neurons during these oscillations highlighted two key phenomena: (1) an asymmetry in the transition from rest to pioneer and reciprocally, leading to a spontaneous increase in the steady proportion of neurons in the excited state, in turn triggering (2) a chain reaction leading all neurons to the excited state provided that the system reaches a sufficient proportion of neurons in that state.

These elements are not specific to the FitzHugh-Nagumo model, but arise in a wide class of excitable systems with noise; we discuss in Sec. V their universality, illustrate the same property in distinct excitable systems, and further discuss the type of transition arising around the synchronized regime.

\section{OSCILLATIONS-INDUCED DESYNCHRONIZATION AND PARKINSON'S DISEASE}

Studying the response of networks of excitable cells in the regime of spontaneous noise-induced oscillations is particularly relevant in the context of Parkinson's disease. Indeed, in Parkinson's disease, spontaneous oscillations emerge in the basal ganglia and motor cortex, and these arise while neurons show an increased excitability $[47,78]$, together with enhanced electrical transmission [49,79]. Some 30 years ago, it was observed that high-frequency stimulation $(\sim 130 \mathrm{~Hz})$ in the basal ganglia relay nucleus, the subthalamic nucleus, had a remarkable effect of alleviating Parkinson's disease symptoms [50,54,57,78,80]. Clinically, the impact of DBS strongly depends on stimulation amplitude and frequency [81]: too-high stimulation frequencies $(>180 \mathrm{~Hz})$ were reported to be therapeutically ineffective $[50,82]$, while too low stimulation frequencies have a still debated impact. Indeed, some studies showed that low-frequency stimulation could worsen some parkinsonian symptoms such as tremors and rigidity [83], possibly by imposing another oscillatory rhythm onto the basal ganglia network, while others showed a beneficial impact of lowfrequency stimulation for gait control and cognitive functions [84]. A comparable dependence on amplitude was reported in patients [85], with too-low stimulations abolishing the improvement of symptoms, sometimes with sudden, thresholdlike loss of efficiency [86]. This observation led to us investigate in more detail the impact of high-frequency stimulation on noise-induced oscillations and the impact of the stimulation parameters.

As shown in Sec. III, the origin of those oscillations is fundamentally stochastic (associated with random transitions between pioneer and resting regimes) and collective (chain reaction), and as such they are distinct in nature from more classical periodic systems. Periodically forced oscillators have a long history in the study of nonlinear dynamical systems, and the complex phenomena associated have been well described: they include phase locking with the stimulus, resonances, phase skipping, and chaos, often associated with the presence of intricate dependencies in amplitude and frequency of the forcing, as the classical 
Arnold tongues (see, e.g., Ref. [87]). Periodic forcing of noise-induced oscillations will induce a distinct phenomenology that we analyze below.

\section{A. Periodic forcing in the noise-induced oscillations regime}

To emulate the impact of DBS on basal ganglia oscillations, we used balanced biphasic waves, typical DBS stimulation profiles advocated by Lilly in the 1960s [88] and widely used clinically. Such stimulations correspond to square waves of positive followed by negative currents with a zero mean:

$$
I(t)=A H\left(\frac{t}{T}\right),
$$

where $A$ is the amplitude of the signal, $T$ is the stimulation period, and $H$ is a periodic profile square wave of period 1 [we will typically use the sign of $\cos (2 \pi t)$ ]. The network equations with DBS stimulation thus read:

$d v_{t}^{i}=\left[f\left(v^{i}\right)-w^{i}+\frac{J}{n} \sum_{j=1}^{n}\left(v^{j}-v^{i}\right)+I(t)\right] d t+\sigma d W_{t}^{i}$,

$d w_{t}^{i}=\varepsilon\left(b v^{i}-w^{i}\right)$.

Extreme regimes of stimulation frequency lead to two expected outcomes: very rapid periodic forcing has almost no impact on the spontaneous oscillations [Fig. 5(a), left], while very slow forcing locks network activity to the periodic signal [Fig. 5(a), right].

Strikingly, for an intermediate value of periodic forcing frequency, the noise-induced oscillations are abolished (at a frequency relatively high compared to the spontaneous noise-induced oscillations frequency). In that antiresonance regime, despite small amplitude oscillations of the voltage and recovery variables in response to the DBS input, we observe a complete absence of collective dynamics or spiking [see Fig. 5(a), center].

Stimulation amplitude also has an important impact: a too-low amplitude $A$ barely affects the spontaneous activity (Fig. 5, bottom). While it could be expected that increasing $A$ would lead the responses of the network to lock to the stimulation, we observed that, at the frequency tested, increasing amplitude did not alter the absence of oscillations for the parameters chosen.

To quantify precisely the DBS amplitude and frequency associated with antiresonance, we computed both the maximal value of the average voltage and recovery variables [Fig. 5(b)] and the maximal value of the Fourier transform of these variables [Fig. 5(c)]. We observed a significant drop both in the amplitude of the average voltage and recovery variable, indicating the absence of collective spiking dynamics in the system, coinciding with a significant drop in the power-spectrum amplitude and frequency, highlighting the loss of synchrony at the network level. This loss of synchrony arises in a relatively wide range of parameter values and for a bounded band of frequency, within a region of parameters $(A, \omega)$ elongated along the amplitude axis. This shows that there exists an optimal stimulation frequency for desynchronization, as soon as the stimulation amplitude exceeds a threshold [89].

\section{B. Microscopic phenomena supporting oscillations-induced desynchronization}

We now discuss the microscopic dynamics supporting this antiresonance phenomenon. To this purpose, we extend the approach proposed for characterizing noise-induced synchronization and describe how the fraction of pioneers $\alpha$ is affected by periodic forcing. The application of a periodic input sweeps neurons back and forth from pioneer to rest and reciprocally, dynamically balancing two opposite phenomena.

(i) During the excitation phase [times for which $I(t)>0$ ], the rate of transition from rest to pioneer increases and the reciprocal rate decreases, neurons become more excitable (the resting state, if it persists under stimulation, gets closer to the quasiseparatrix), and $\alpha_{c}$ decreases. Consequently, neurons accumulate faster within the pioneer regime, and may trigger a spike faster than in the unperturbed system.

(ii) During the inhibition phase [times for which $I(t)<0]$, pioneers are rapidly brought back near the rest state.

Because of these dynamical fluctuations of transition rates, the period of stimulation $T$ is crucial for antiresonance. While a long inhibition phase would indeed prevent neurons from firing, the biphasic balanced stimulations profile will in turn present a long (or large amplitude) excitation phase during which one or multiple spikes may be fired. In contrast, a too rapid signal will not allow enough time during the inhibition phase to drive the pioneers back to rest, leading to a progressive accumulation of neurons in the pioneer state and eventually to a macroscopic spike, with the largest frequencies having no impact on the period. At intermediate frequencies, the two phenomena may balance and jam the system below the chain reaction threshold. For this to occur, the period of the stimulation should be smaller than the time it takes for the system to reach the chain reaction threshold when the input is $+A$, but not much smaller so as to allow a proper compensation during the inhibition phase. A stimulation frequency higher than the spontaneous oscillation frequency is needed to prevent the emergence of synchrony, in line with DBS in Parkinson's disease.

Quantitatively characterizing the antiresonance phenomenon requires keeping track of neurons continuously switching from rest to pioneer. We thus extended the simple resting-pioneer model to include transitioning neurons: 
(a) Network example responses to periodic forcing
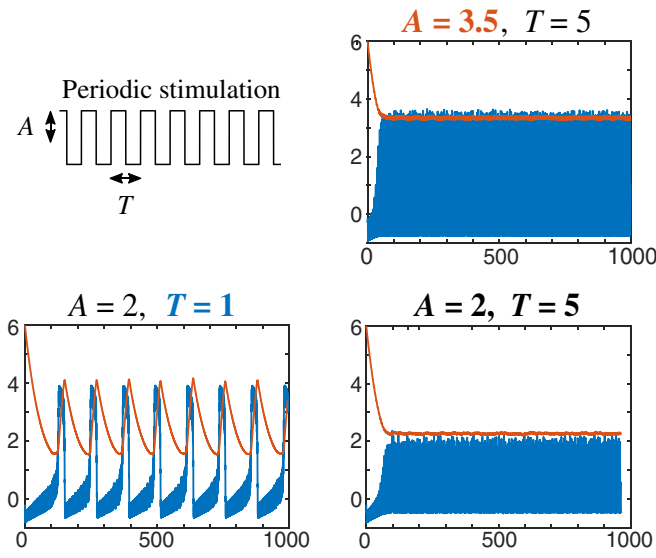

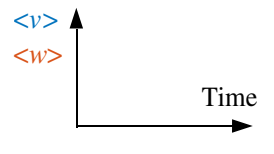

(b) Maximal responses to periodic forcing
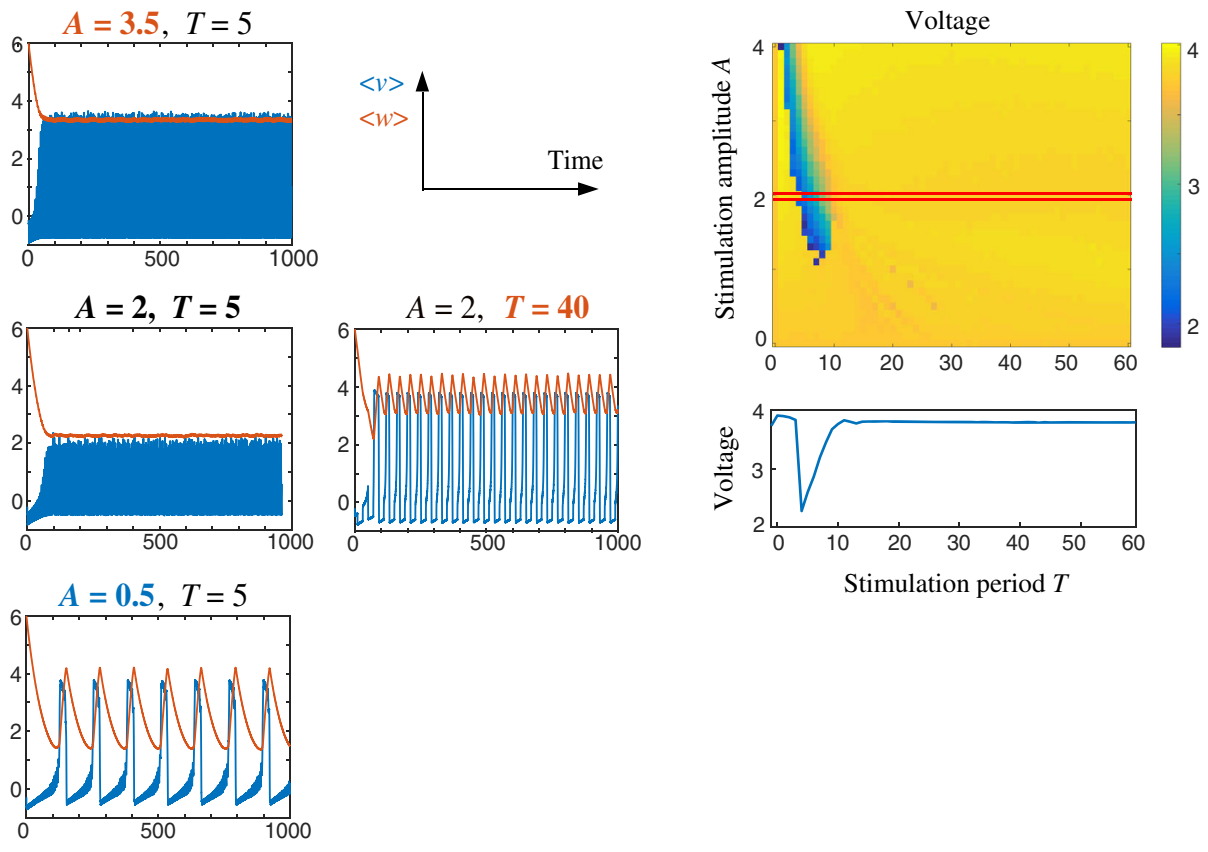

(c) Fourier analysis of the average recovery variable to periodic forcing
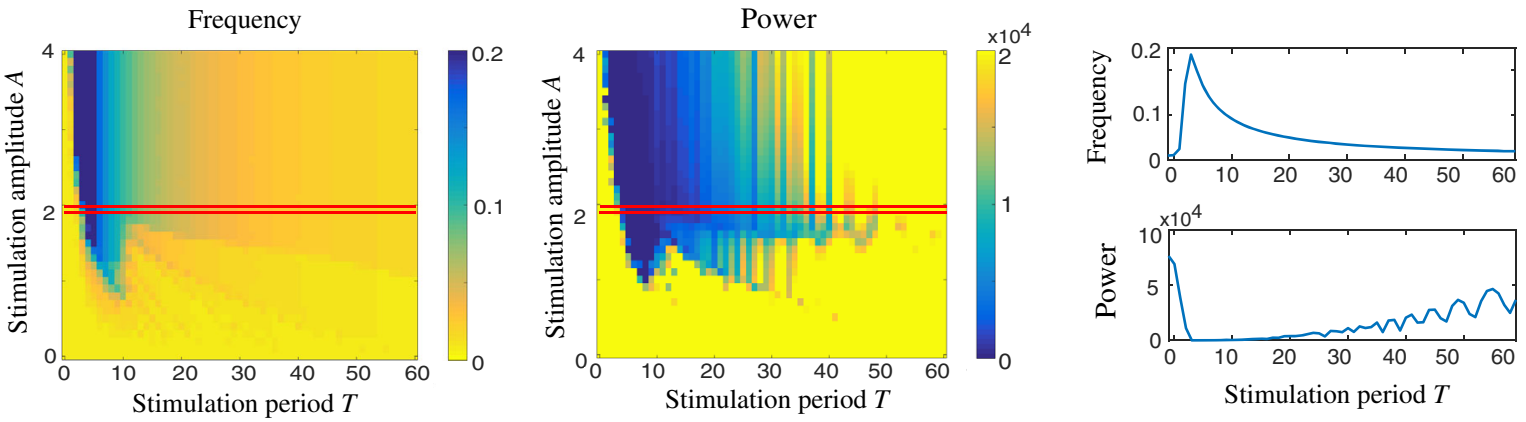

FIG. 5. Desynchronization induced by high-frequency periodic stimulation of the Fitzhugh-Nagumo network equation (with same parameters used in Fig. 1 and with $J=1.5$ and $\sigma=1.5$ ) for various values of the amplitude $A$ and period $T$ of the stimulation current. (a) Trajectories and desynchronization. Dynamics of the average voltage (blue) and recovery variable (red). The system becomes desynchronized for relatively high-frequency stimulation $(T=5)$, once the amplitude of the stimulation becomes strong enough to perturb the system. Increasing further the stimulation frequency leads to the return of intrinsic oscillations $(T=1)$, while slow periodic forcing induces a phase locking with the stimulation $(T=40)$. (b) Top: The maximal value of the average voltage clearly delineates a region of desynchronization (in blue), corresponding to a dip in the maximal average as visible for fixed amplitude (bottom graph, corresponding to the red slice in the upper diagram). (c) Preferred frequency and maximal power of the Fourier transform of the average recovery variable delimitates regions of synchronization (yellow), desynchronization (dark blue), and period skipping, with sections at fixed amplitude (red slices in the heat maps and right-hand panels).

instead of instantaneous transitions from rest to pioneer, neurons that cross the quasiseparatrix enter a transitioning state, before gradually turning into pioneers in a time evaluated as the typical time of transition [see Fig. 6(a)]. Stochastic transitions from rest to transitioning neuron and from pioneer to rest occur as characterized in the previous section, and the rates now depend on time following the fluctuations of the input $I(t)$. In addition to these stochastic transitions, deterministic transitions from pioneer to rest through spiking, and from transitioning neurons to pioneers according to the flow, occur at typical times respectively denoted $T_{s}$ and $\tau$.

In the periodically forced system, the resting, transitioning, and pioneer states are relative to the value of the input. Moreover, in the stochastic network system, the population of neurons do not have homogeneous voltages (in particular because of noise and continuity of the trajectories). This variation in voltage can no longer be neglected for the 
antiresonance phenomenon. For instance, when the input switches from $+A$ to $-A$, a fraction of transitioning neurons (including in particular those that have just switched from rest) will have low voltages within the resting range of the system with $I=-A$, and a fraction of neurons from the pioneer population will belong to the transitioning range of voltage. The longer a transitioning neuron has been in the transitioning state (with, say, $I=+A$ ), the larger its voltage, and thus the less likely it will become a resting neuron when the input switches to $-A$. Similarly, when the input switches from $-A$ to $+A$, some resting neurons become transitioning and some transitioning neurons become pioneers.

We developed a simple toy model recapitulating these phenomena in a two-dimensional equation, describing the fractions of (i) resting neurons, that did not start their transition, (ii) transitioning neurons, that initiated a transition to pioneers, and (iii) pioneers. Respective proportions in each state are denoted $\alpha_{R}(t), \alpha_{T}(t), \alpha_{P}(t)$ [with, for all times, $\left.\alpha_{R}(t)+\alpha_{T}(t)+\alpha_{P}(t)=1\right]$, and evolve according to the following dynamics [see Fig. 6(a)].

(i) A resting neuron becomes a transitioning neuron with rate $K_{R P}\left[I(t), \alpha_{P}(t)\right]$ [transition rate with making explicit the dependence in $I$, solid pink arrow in Fig. 6(a)].

(ii) A transitioning neuron becomes pioneer at rate $1 / \tau$ [solid blue arrow in Fig. 6(a)].

(iii) A pioneer neuron returns to rest due to noise with rate $K_{P R}\left[I(t), \alpha_{P}(t)\right]$, and after spiking with a rate $1 / T_{s}$ [orange arrow in Fig. 6(a)].

(iv) When the input switches from $I=+A$ to $-A$, a proportion $S_{+}(T / \tau)$ of transitioning (pioneer) neurons become resting (transitioning) neurons, with $S_{+}$ a decreasing sigmoid with $S_{+}(0)=1$ and $S_{+} \rightarrow 0$ at infinity [dotted orange and purple arrows in Fig. 6(a)].

(v) When the input switches from $I=-A$ to $+A$, a proportion $1-S_{-}(T / \tau)$ of transitioning (resting) neurons become pioneer (transitioning) neurons, with $S_{-}$a decreasing sigmoid with $S_{-}(0)=1$ and $S_{-} \rightarrow 0$ at infinity [dot-dashed pink and blue arrows in Fig. 6(a)].

Therefore, during periods of constant input, the fractions of pioneers, resting, and transitioning neurons satisfy the equations:

$$
\begin{aligned}
& \dot{\alpha}_{P}=-\alpha_{P} K_{P R}\left[I(t), \alpha_{P}(t)\right]+\frac{\alpha_{T}}{\tau}-\frac{\alpha_{P}}{T_{s}}, \\
& \dot{\alpha}_{T}=\alpha_{R} K_{R P}\left[I(t), \alpha_{P}(t)\right]-\frac{\alpha_{T}}{\tau}, \\
& \alpha_{R}=1-\alpha_{P}-\alpha_{T},
\end{aligned}
$$

together with the jumps when the input switches, (a) Simplified model

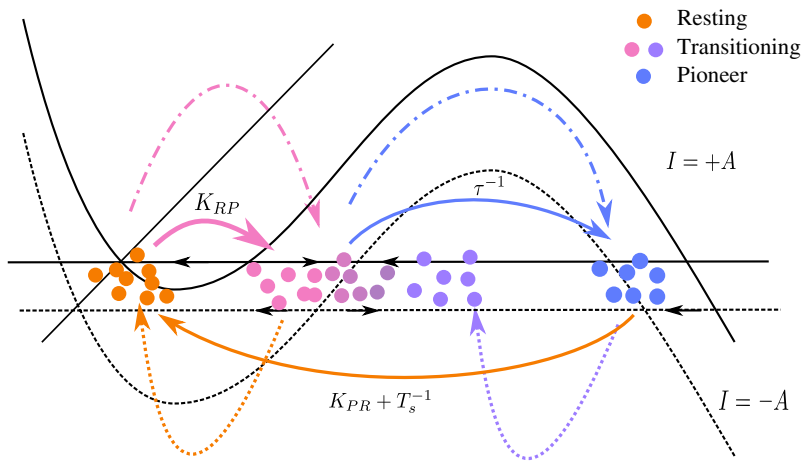

(b) Simulations of the different regimes
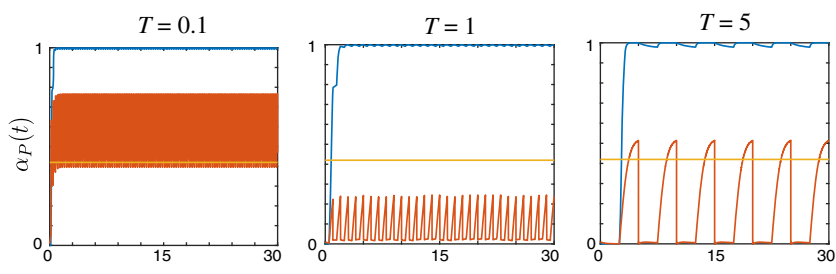

FIG. 6. Principle of the model of oscillations-induced desynchronization. (a) Phase plane of the toy model, with nullclines for $I=A$ (solid line) or $I=-A$ (dotted line), together with the three populations of cells: resting (orange), transitioning (pink to violet according to the value of $v$ ), and pioneer (blue), together with the transitions at fixed input (solid lines) and at switching times from $+A$ to $-A$ (dashed lines) or from $-A$ to $+A$ (dot-dashed lines). (b) Simulation of the proportion of pioneers in the rest-pioneer model (blue) and in the three-population model (red) as a function of time for increasing stimulation period. No qualitative change is observed in the rest-pioneer model (blue lines): for any frequency, a chain reaction is predicted. In the three-population rest-transitioning-pioneer model, we recover the phenomenon of Fig. 5. Left: For rapid oscillations, compensation of the pioneers does not occur, and the system eventually exceeds $\alpha_{c}$ (yellow line). Middle: Optimal oscillation frequency. The system stabilizes around the critical value with a mean below that value preventing spiking. Right: Slow oscillations synchronize the system to the input. In that model, $K_{R P}( \pm A, \cdot)$ and $K_{P R}( \pm A, \cdot)$ were computed numerically as in the previous section, with $A=2, \tau=2$ and the probability to switch is a sigmoidal (tanh) function of $(\tau \omega)^{-1}$ with slope 4 and threshold 0.2 , and the periods of stimulation chosen are, from left to right, $T=0.1,1,5$.

$$
\begin{aligned}
& (I:-A \rightarrow+A)\left\{\begin{array}{l}
\alpha_{P}=\alpha_{P}+S_{+}\left(\frac{T}{\tau}\right) \alpha_{T}, \\
\alpha_{T}=S_{+}\left(\frac{T}{\tau}\right) \alpha_{R}+\left[1-S_{+}\left(\frac{T}{\tau}\right)\right] \alpha_{T}
\end{array},\right. \\
& (I:+A \rightarrow-A)\left\{\begin{array}{l}
\alpha_{P}=S_{-}\left(\frac{T}{\tau}\right) \alpha_{P}, \\
\alpha_{T}=S_{-}\left(\frac{T}{\tau}\right) \alpha_{T}+\left[1-S_{-}\left(\frac{T}{\tau}\right)\right] \alpha_{P} .
\end{array}\right.
\end{aligned}
$$

We numerically simulated this simple system of ODEs with jumps using the rates of transitions and spike duration 
computed in Sec. III and found that it accurately captures all observations associated with the oscillations-induced desynchronization [Fig. 6(b)]. In particular, we recover the nonmonotonic dependence in stimulation frequency. At high frequency, the input plays a minor role: resting neurons progressively transition to pioneer, and neurons end up firing collectively, since the too brief inhibition phase is insufficient to counterbalance the accelerated transition of resting neurons to the pioneer state [Fig. 6(b), left]. At low frequency, macroscopic spikes are fired, as visible in the pioneer fraction exceeding the critical fraction [Fig. 6(b), right]. However, intermediate frequencies can finely balance the transitions to the pioneer state and stabilize the fraction of pioneers below the critical fraction $\alpha_{c}$. In that case, a majority of neurons end up fluctuating within the transitioning regime, and the fraction of pioneers does not reach the critical fraction associated with the chain reaction [Fig. 6(b), middle].

This model allows a deeper understanding of some features of the oscillation-induced desynchronization. Starting from a regime of noise-induced synchronization, it is clear that to prevent any firing, a necessary condition is that the input is large enough for the system with input $-A$ to be clamped to rest. Indeed, our three-variable model alternates tracking the high and low equilibria of $\alpha_{P}$, denoted $\alpha^{+}$and $\alpha^{-}$as $I$ switches from $A$ to $-A$. At low stimulation amplitude, $\alpha^{+}$and $\alpha^{-}$are close to the equilibrium value of $\alpha$ in the absence of input, and therefore both fractions will be above $\alpha_{c}$ for sufficiently low amplitude. In that case, no desynchronization will occur, as was observed in the full stochastic system [Fig. 5(b)]. Moreover, a decreasing relationship between $A$ and $\omega$ was observed in the regime of desynchronization. From the simplified model viewpoint, this relationship can be interpreted noting that, for larger input amplitudes, the proportion of pioneers will more quickly increase above $\alpha_{c}$, requiring faster switches to stabilize below $\alpha_{c}$.

\section{Information transmission}

Stochastic excitable systems thus reproduce the alteration of spontaneous oscillations in the presence of highfrequency stimulation as observed in Parkinson's disease. Taking the parallel with Parkinson's disease one step further and the oscillation-induced desynchronization regime analogy with the therapeutic effect of DBS, we investigated the possible impact of periodic stimulation on the transmission of information of the network. Indeed, DBS has been associated with the restoration of normal activity patterns in the basal ganglia and a decrease in motor symptoms in parkinsonian patients, as discussed above.

In the stochastic system, when oscillations abolish collective noise-induced desynchronization, the system is maintained in a dynamical state balancing the natural tendency of the system to fire in the noise-induced oscillations regime and keeping the system on the verge of a chain reaction. Remaining within a highly reactive state, the stochastic network may thus be able to respond rapidly and precisely to external stimulations. This is indeed what we can visually observe in Fig. 7(a), showing a strong modification of the response of the network to a white noise stimulus for the parameters associated with the interruption of oscillations (center) in the average voltage traces in the desynchronized regime and a reduced dependence on the periodic DBS forcing pattern. This situation contrasts with the regimes in which intrinsic or forced high-amplitude oscillations dominate even in the presence of the stimulus.

To test this hypothesis, we estimated the capability of the periodically forced FitzHugh-Nagumo stochastic network to transmit information from a current injected to all neurons in the network. Computing information in a spiking network is a complex task, particularly when considering that information is contained in the times of the spikes, that are discrete events [90]. Here, for simplicity, we computed the information contained in the averaged voltage. In detail, we computed the Pearson's correlation coefficient between the averaged voltage of the network and the stimulus. This quantity, formally defined as the covariance of the averaged voltage and the input divided by the product of their standard deviations (thus, belonging to the interval $[-1,1]$ ) provides a linear estimate of the relationship between input and the averaged output. A larger Pearson's correlation coefficient indicates that the two variables are linearly correlated; i.e., they have a tendency to fluctuate simultaneously, in the same direction. Figure 7(b) reports the result of the correlation analysis for 4 distinct stimuli, arranged from least to most regular: a white noise, an Ornstein-Uhlenbeck process, an OrnsteinUhlenbeck centered at a cosine, and a pure cosine. While the amplitude of the Pearson's correlation coefficient is very distinct between all 4 cases (it increases with the regularity of the input), a similar profile tends to emerge with a peak arising in the region of DBS parameters associated with an abolition of oscillations. This is visible in Fig. 7(d), where we plotted relevant level sets for Pearson's coefficients for each stimulus versus a level set associated with the abolition of oscillations [maximal average voltage, as in Fig. 5(b)]. Moreover, we computed the correlation coefficient between the Pearson's correlation coefficient map (our estimate of the information, peaking in regions of efficient information transmission) and the maximal voltage amplitude of variation (dropping when oscillations are abolished). We found strong anticorrelations between the two variables, showing clearly that information peaks when oscillations disappear (white noise, $-61.9 \%$; Ornstein-Uhlenbeck, $-62.3 \%$; OrnsteinUhlenbeck centered at a cosine, $-62.9 \%$; cosine, $-56.7 \%$ ), meaning that the peak in information transmission corresponds to parameters associated with low-amplitude 
(a) Network responses to stimulus under periodic forcing
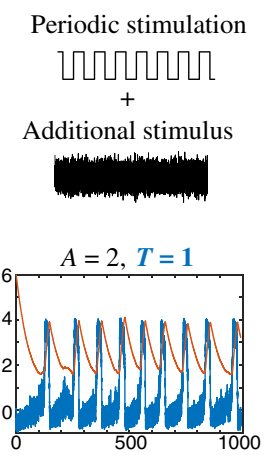
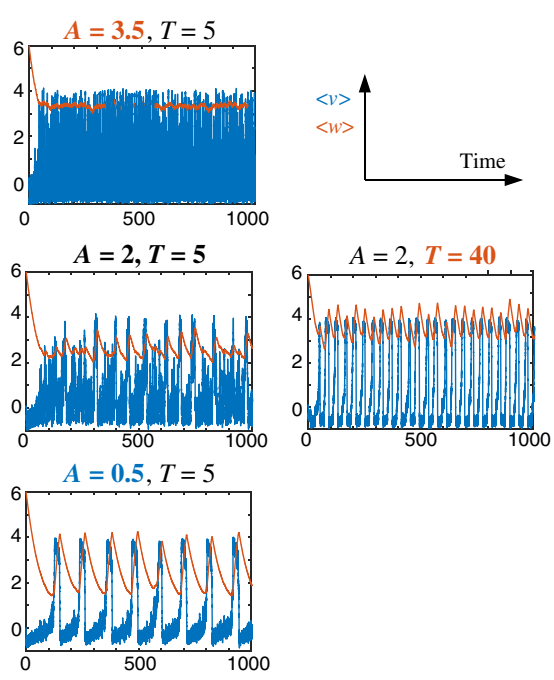

(b) Information capacity
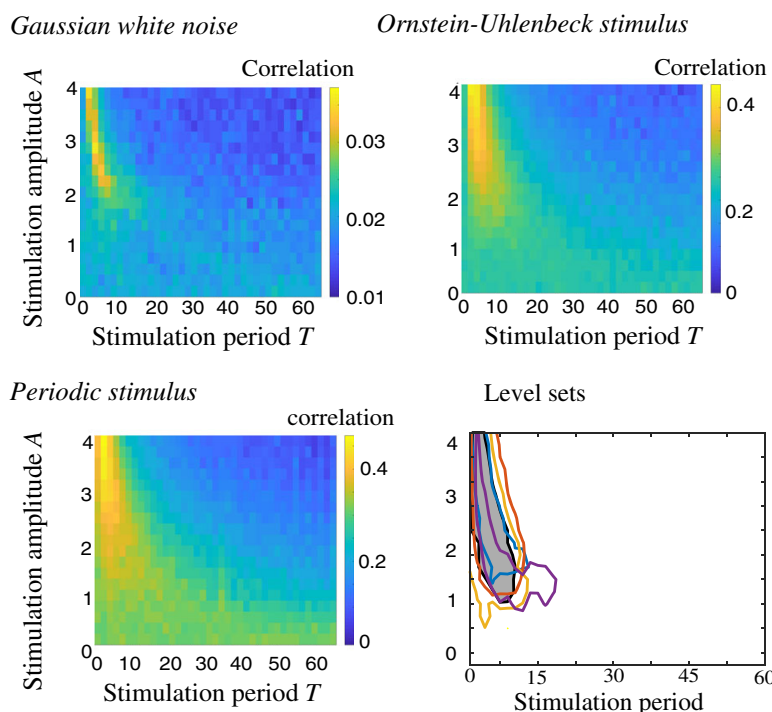

Level sets

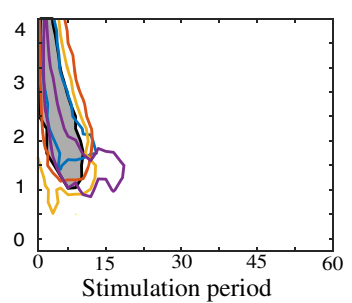

(c) Renormalized correlations
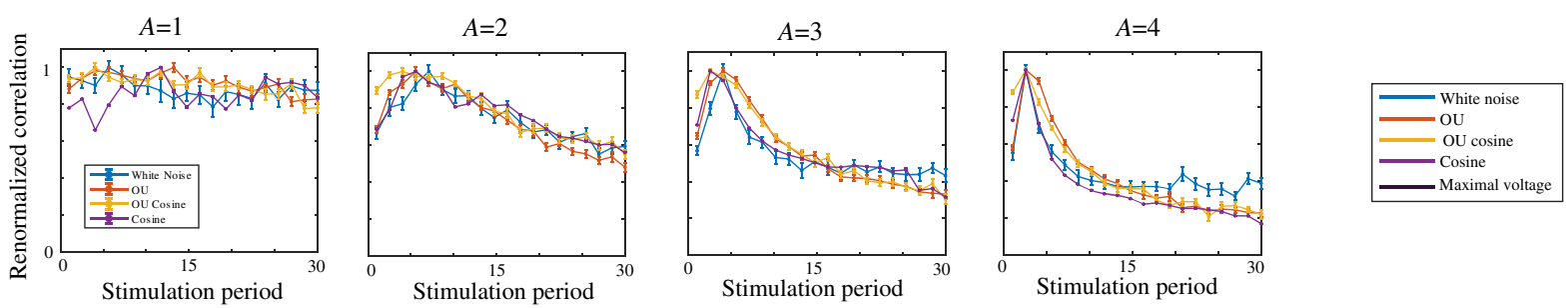

FIG. 7. Information transmission of the Fitzhugh-Nagumo network model evaluated by Pearson's correlations between stimulus and network response. Parameters as in Fig. 6(a) (oscillations-induced desynchronization, with $n=4000$ neurons). (a) Trajectories of mean network activity in response to Gaussian white noise, as a function of DBS parameters (amplitude and frequency). (b) Pearson's correlation coefficient between average voltage and input for various values of the amplitude $A$ and period $T$ of DBS for Gaussian white noise input (upper left), stationary Ornstein-Uhlenbeck (OU) process (upper right), and Ornstein Uhlenbeck process centered at a sine wave (lower left). Lower right-hand panel shows a level set for each of these maps (blue, white noise; red, Ornstein-Uhlenbeck; yellow, Ornstein-Uhlenbeck centered at a sine wave; purple, pure cosine) compared with the level set associated with maximal voltage amplitude, identifying the loss of spontaneous oscillations in Fig. 5(b). (c) Rescaled Pearson's coefficient as a function of DBS period for 4 values of the input amplitude, as a function of DBS period, show nicely overlapping profiles indicating consistent improvement of the information process for all types of stimuli depending on stimulus.

responses, i.e., with the absence of oscillations. Moreover, we observed that while the amplitude of correlations differed significantly between stimuli, the relative variation (computed as the Peason's coefficient divided by the maximal value for a given DBS amplitude) yielded almost identical profiles of dependence upon DBS frequency for all stimuli tested, arguing for the fact that these responses are properties of the network rather than stimulus specific [Fig. 7(c)].

We confirmed these results in Appendix using mutual information, an alternative statistical measure quantifying the amount of information one can obtain from the stimulus by observing the network output in response to that stimulation. These analyses precisely confirmed the conclusions drawn from the correlation analyses. Overall, these results suggest that highly stable intrinsic or forced oscillations limit stimulus information encoding, while their destabilization through high-frequency stimulation endows the system with more computational capabilities, and that the gain in the efficiency of information transmission is maximized for appropriate stimulation amplitude and frequency. This disruption in information transmission depending on frequency of external signals is also evocative of recent theoretical observations reporting how specific frequencies can disrupt persistent states in working memory [91].

\section{UNIVERSALITY}

We now study the universality of the noise-induced synchronization and antiresonance in a class of interacting excitable elements, as well as the existence of universal transitions by which synchrony emerges from clamping or 
asynchrony. We give further evidence of this universality by presenting numerical simulation of three other stochastic neural network models and bifurcation analyses.

\section{A. How do noise-induced oscillations emerge and disappear?}

\section{Noise-induced oscillations}

Section III has shown, in the FitzHugh-Nagumo model, that noise-induced oscillations were the result of the buildup of an imbalance in the stochastic transition rates of neurons between the resting state and an excited state eventually leading to a chain reaction, and that this phenomenon could be described by a simple one-dimensional birth-and-death Markov chain (or its ODE counterpart) tracking the fraction of cells in each state. This phenomenon relies on the following few crucial elements: (1) excitability, accounting for the transitions between rest and an excited state, and (2) confininglike interactions (e.g., diffusive coupling), preserving the coherence of the elements.

For excitable systems with such interactions, we conjecture that one will recover both elements leading to noiseinduced oscillations. In particular, for such systems, the existence of a critical fraction of excited neurons above which a synchronized spike occurs will be ensured provided that the coupling strength is sufficiently large to prevent individual spikes. Indeed, given a particle near the resting state, the presence of a large fraction of excited neurons and confining interactions will act as a driving force counterbalancing the stability of the resting state, and, for coupling or $\alpha$ large enough, will lead to a destabilization of the resting state. Moreover, in such systems, a proper amount of noise will naturally lead the system to exceed that critical fraction of excited neurons. Indeed, the transition rates from rest to the excited state and reciprocally are generally asymmetric in excitable systems. This asymmetry could be associated to the fact the excited state is not a stable equilibrium of the dynamical system, but a transient state preceding a large excursion away from the resting state, while the dynamics near the resting state are stationary. In other words, excited elements are driven to a remote state and less likely to return to rest than elements near rest that have a vanishing vector field. This should naturally lead to an imbalance in the rates of transition: once a neuron has transitioned to the excited state, it will likely have a smaller rate of transition to rest than the reciprocal rate. Moreover, confining interactions will naturally amplify this imbalance. For this collective phenomenon to build up, a sufficiently strong coupling is necessary to provide coherence to the set of neurons, but a too large coupling will limit the transitions, clamping the system at rest. Therefore, we conclude that stochastic networks of excitable systems should present noise-induced oscillations for a limited range of coupling strengths allowing sufficient coherence to the set of elements, yet sufficient flexibility to allow the buildup of the chain reaction. Noise levels should also be limited, as observed in the FitzHugh-Nagumo network: (1) too little noise leads to rare transitions, and because of the natural return of each element to rest, the critical proportion of excited elements is never reached, and (2) too much noise, making the transition rates from rest to pioneer and reciprocally more symmetric and breaking the excitable structure of the intrinsic dynamics, will prevent any significant increase in the fraction of excited elements.

These phenomena are thus not specific to the FitzHughNagumo network, and should be valid in a broad class of excitable systems with synchronizing coupling.

\section{Transitions to and from noise-induced oscillations}

As observed in the FitzHugh-Nagumo network for a given coupling level, three regimes arise as noise is increased: clamping near the resting state, oscillations, and asynchrony. The nature of the transitions to and from the oscillatory regime can also be inferred from the analysis of each neurons' dynamics, and will thus share the same universality properties.

At low noise, the rarity of transitions between rest and excited states prevents the system from reaching the critical proportion of excited elements, and thus, in the long run, the system reaches a steady proportion of excited elements below that critical threshold. As noise is increased, transition rates from rest to pioneer and reciprocally will increase asymmetrically, leading to a progressive increase in the steady proportion of pioneers, until that proportion reaches the critical fraction associated with the chain reaction. In the vicinity of this transition and within the clamping regime, the proportion of pioneers asymptotically tangents a level slightly below the critical fraction, and as the transition is crossed, it takes an arbitrary long time to trigger a synchronization event; moreover, this event will be massively synchronized because of the relatively low level of noise. Therefore, as noise is slowly increased from the clamped regime, the collective dynamics suddenly transitions from a stationary to arbitrary slow, large amplitude, highly synchronized oscillations. This type of dynamics is evocative of homoclinic bifurcations, and we conjecture that excitable networks displaying noiseinduced transitions switch to these oscillations through a collective homocliniclike bifurcation.

When noise is further increased, oscillations will reach a finite frequency associated with the deterministic time needed for the population of excitable elements to reach the chain reaction threshold, while losing progressively coherence due to an increased influence of independent fluctuations. This loss of coherence will lead to a decrease in the average voltage oscillation amplitude, while the period of the oscillations remains lower bounded by the typical time of an excursion. Therefore, as noise is increased, oscillations will progressively disappear through a desynchronization evocative of a supercritical Hopf bifurcation. 
This explanation, perfectly in line with the numerical evaluation of the mean and period of the oscillation in the FitzHugh-Nagumo network in Fig. 1, will be confirmed in several excitable systems in Sec. V B. Moreover, we will verify the presence of the conjectured transitions in the two simple models (theta neuron and Wilson-Cowan models) for which one can access the bifurcation diagram of the probability distribution.

\section{Oscillations-induced desynchronization}

In the class of excitable systems showing noise-induced oscillations, we further tested the impact of high-frequency periodic forcing and found again that the phenomenon enjoys a relatively broad universality. The microscopic dynamics supporting the phenomenon uncovered in the FitzHugh-Nagumo network in the previous section indeed calls upon relatively general mechanisms resulting in neurons remaining dynamically within a transitioning regime whereby neurons are neither at rest nor fully in the excited regime: positive phases of the input, while they may accelerate the emergence of a chain reaction, are quickly compensated by the negative phases of the input. This general phenomenon requires, however, a relatively slow transition from rest to pioneer, which is not always found in simple models. We will recover this desynchronization in other models in Sec. V B.

\section{B. Exploring the universality class}

To confirm the universality of both transitions, we simulated three other networks of excitable elements with noise, in the large $n$ limit, as coupling and noise are varied.

\section{Morris-Lecar model}

We start considering the stochastic, electrically coupled network of Morris-Lecar neurons [92], a classical biophysically realistic neuron model particularly interesting for its relative simplicity yet direct relationship with electrophysiology and with the Hodgkin-Huxley model. In this model, the state of neuron $i \in\{1, \ldots, n\}$ is described by a voltage variable $v^{i}$ and a recovery $w^{i}$ whose dynamics are governed by the equations:

$$
\begin{aligned}
d v_{t}^{i}= & \frac{1}{c}\left[I-g_{C a}\left(v^{i}-v_{C a}\right) m_{\infty}\left(v^{i}\right)-g_{K}\left(v^{i}-v_{K}\right) w\right. \\
& \left.-g_{L}\left(v-v_{L}\right)+I+J\left(\langle v\rangle-v^{i}\right)\right] d t+\sigma d W_{t}^{i}, \\
d w_{t}^{i}= & \frac{\phi}{\tau_{w}\left(v_{t}^{i}\right)}\left[w_{\infty}\left(v_{t}^{i}\right)-w_{t}^{i}\right],
\end{aligned}
$$

with $\langle v\rangle(t)=(1 / n) \sum_{j=1}^{n} v_{t}^{j}$. In that model, $c$ denotes the membrane capacitance, $I$ is a current, $g_{L}, g_{K}, g_{C a}$ are the leak, $\mathrm{K}^{+}$and $\mathrm{Ca}^{2+}$ conductances through membrane channels, $v_{l}, v_{K}, v_{C a}$ their reversal potentials, $m_{\infty}(v)$ accounts for an instantaneous calcium current, $\phi$ is a reference frequency, $\tau_{w}$ the timescale of recovery $w$, and $w_{\infty}$ is the quasisteady-state value of $w$. We refer to Chap. 3.2 of Ref. [6] for the specific sigmoidal shapes of $\tau_{w}, m_{\infty}$, and $w_{\infty}$ as well as for basic parameter values. The above equation incorporates noisy currents driven by independent Brownian motions $\left(W_{t}^{i}\right)$ and diffusive coupling modeling electrical synapses.

It is well known that this system is excitable within a wide range of parameter values [6]. We thus analyzed the impact of noise and electrical coupling within this excitability regime, and recovered the noise-induced oscillation transition, similar in many ways to the observations made in the FitzHugh-Nagumo network [see Fig. 8(a)]. In particular, at high coupling or low noise, the system is clamped in the vicinity of the resting state [left and top diagrams in Fig. 8(a1)], while at low coupling or large noise, asynchronous dynamics take over [bottom and right diagrams in Fig. 8(a1)]. Between these two regimes, noise induces perfectly periodic synchronized oscillations. The type of transitions is also clearly recovered: for a fixed level of coupling, sharp and large amplitude, arbitrarily slow oscillations appear suddenly as noise is progressively increased, again evocative of a homoclinic transition, and, as noise is further increased, these oscillations progressively lose synchrony, as visible in the gradual decrease in the amplitude of the average voltage at reaching the asynchronous regime. These observations are also visible in the Fourier analysis associated.

Furthermore, the noise-induced oscillations were found to disappear under application of a biphasic Lilly pulse $I(t)=A H(t / T)[$ Eq. (8)] for periods and amplitudes within specific bounds: too rapid oscillations do not affect the spontaneous oscillatory dynamics [Fig. 8(a2), left], too slow oscillations lock the system to the stimulus [Fig. 8(a2), right], and appropriate frequency and amplitude abolish the synchronization [Fig. 8(a2), center], a phenomenon arising in a relatively broad range of stimulation periods and amplitudes as shown in the Fourier analysis presented in the lower left-hand panel of Fig. 8(a2).

\section{Electrically coupled theta neuron network}

The theta neuron constitutes a canonical example of excitable system [6]. The stochastic electrically coupled theta neuron system describes the phase of neuron $i$ in an $n$-neurons network as a variable $\theta^{i} \in \mathbb{S}^{2 \pi}$ (the onedimensional torus $\mathbb{R} / 2 \pi \mathbb{Z}$ ) through the equations:

$$
\begin{aligned}
d \theta_{t}^{i}= & \left(1-\cos \left(\theta_{t}^{i}\right)+\left[1+\cos \left(\theta_{t}^{i}\right)\right]\left\{-a-\sigma^{2} \sin \left(\theta_{t}^{i}\right)\right.\right. \\
& \left.\left.+\frac{J}{n} \sum_{j=1}^{n}\left[q\left(\theta_{t}^{j}\right)-q\left(\theta_{t}^{i}\right)\right]\right\}\right) d t+\sigma\left[1+\cos \left(\theta_{t}^{i}\right)\right] d W_{t}^{i},
\end{aligned}
$$

with $q(\theta)=[\sin (\theta) / 1+\cos (\theta)+\varepsilon]$ for $\varepsilon$ small (in our numerical simulations, $\varepsilon=0.001)$. Classical theory of mean-field limits ensures that, for $n \rightarrow \infty$, the probability 
(a) Morris-Lecar model

(a1) Noise-induced oscillations

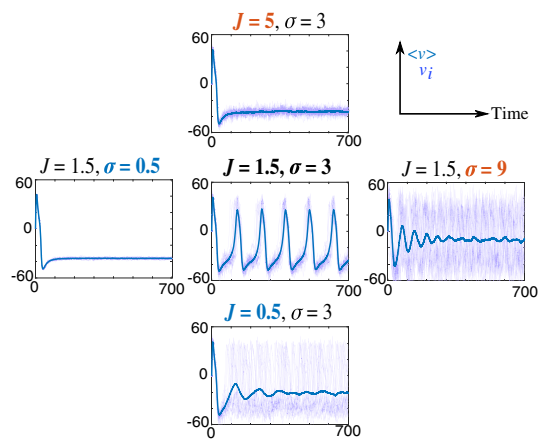

(b) Bifurcation diagram of the theta model

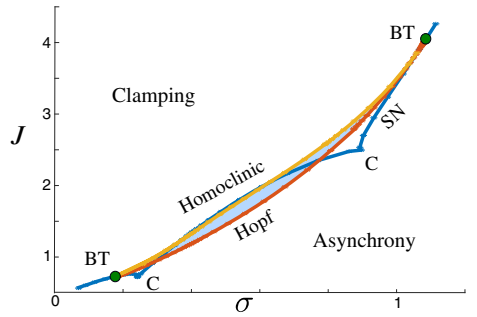

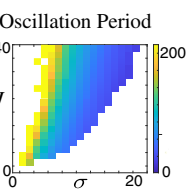

Fourier Amplitude

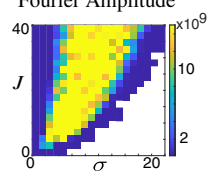

(a2) Oscillations-induced desynchronizatio $\mathrm{n}$

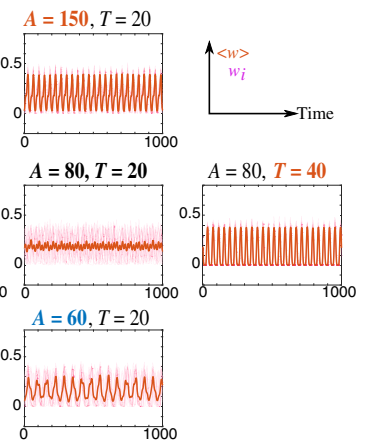

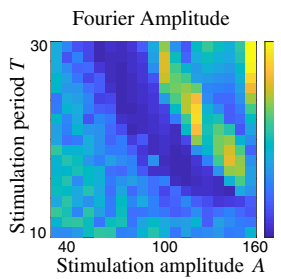

(c) Wilson-Cowan model
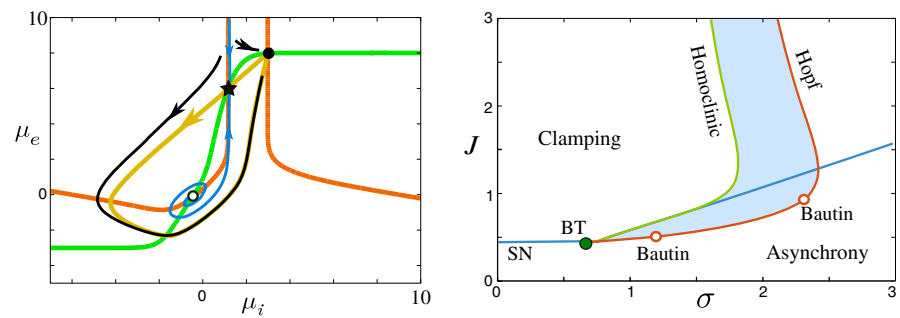

FIG. 8. Universality of the transitions. (a) Morris-Lecar model. Noise-induced transition and oscillations-induced desynchronization; parameters as in Table 3.1 of Ref. [6] (SNLC), with $\phi=0.02, V_{1}=-1.3, V_{4}=10$, and basic applied current $I=35$. (a1) shows the noise-induced synchronization phenomenon as a function of coupling strength $J$ and noise $\sigma$. Trajectories, 20 sample voltage traces (dark blue) and mean (bold cyan); heat maps, statistics of the synchronization via Fourier transform peak period (top) and amplitude (bottom) as a function of coupling strength and noise. (a2) Responses of the system within the noise-induced oscillations regime $(J=1.5, \sigma=3)$ to a Lilly pulse Eq. (8) for various frequencies and amplitudes; at appropriate frequency and amplitude, oscillations disappear (center). Trajectories, 20 sample recovery variable traces (red) and mean (bold black); heat map, amplitude of the Fourier transform as a function of period and amplitude of the stimulation. (b) The theta neuron with $a=0.04$. Two-parameter bifurcation diagram of the Fokker-Planck equation of the theta neurons as a function of coupling strength and noise shows a saddle-node bifurcation manifold (blue) and a Hopf bifurcation manifold (red), together with two codimension-two cusp and two Bogdanov-Takens (BT) bifurcations, associated with a branch of saddle-homoclinic bifurcation (yellow). The Hopf and homoclinic bifurcation curves delineate an eye-shaped noise-induced synchronization regime (sky blue region). (c) The excitatory-inhibitory Wilson-Cowan mean-field system with $g_{e e}=15, g_{e i}=-12, g_{i e}=16, g_{e e}=-5, I_{e}=0, I_{i}=-3, S(x)=\operatorname{erf}(3 x)$. The system exhibits an intrinsic excitable structure (left) with a single stable fixed point (black circle) and a saddle fixed point (black star) whose stable (blue) and unstable (yellow) manifolds organize the excitability. Green and red curves are the nullclines of the system. Right: Two-parameter bifurcation diagram of the excitatory-inhibitory Wilson-Cowan system features a Hopf (red solid line, supercritical; red dashed line, subcritical; red circle, codimension-two Bautin bifurcation) and a saddle-node (blue) bifurcation colliding at a Bogdanov-Takens bifurcation (green circle), from which point emerges a homoclinic bifurcation (green line). Near the BT point, the saddle-node bifurcation shows a cusp (not visible in the diagram). The homoclinic and Hopf bifurcations delineate a region of noise-induced oscillations (blue) that extends slightly beyond the Hopf curve between the two Bautin bifurcations and disappear through a fold of limit cycles (not shown). As noise is increased, oscillations emerge from clamped regimes through the homoclinic bifurcation and disappear through the Hopf bifurcation (or the fold of limit cycles) into an asynchronous state.

distribution $p(t, \theta)$ of any given neuron in the network to be at phase $\theta$ at time $t$ converges to the solution of the nonlocal equation:

$$
\begin{aligned}
\partial_{t} p= & -\partial_{\theta}\left[p \left(1-\cos (\theta)+[1+\cos (\theta)]\left\{-a-\sigma^{2} \sin (\theta)\right.\right.\right. \\
& \left.\left.\left.+J\left[\int_{0}^{2 \pi} q\left(\theta^{\prime}\right) p\left(t, \theta^{\prime}\right) d \theta^{\prime}-q(\theta)\right]\right\}\right)\right] \\
& +\frac{\sigma^{2}}{2} \partial_{\theta}^{2}\left\{p(t, \theta)[1+\cos (\theta)]^{2}\right\} .
\end{aligned}
$$

Numerical simulations of the stochastic network equation as well as the mean-field Fokker-Planck equation recover the emergence of synchronized oscillations for appropriate coupling and noise levels. Here, instead of presenting numerical simulations of the network equation, we computed the bifurcations of the mean-field equation (9), thus finding the precise boundaries of the synchronization regime.

To this end, we discretized this equation for $\theta \in\left\{\theta_{k}=\right.$ $\left.\left(k 2 \pi / N_{\text {grid }}\right), k=0, \ldots, N_{\text {grid }}-1\right\}$ with $N_{\text {grid }}=100$, thus replacing the nonlocal partial differential equation into an 
$N_{\text {grid }}$-dimensional ordinary differential equation describing the probabilities $p_{k}(t)=p\left(t, \theta_{k}\right)$, similar to the system written above, with the operator $\partial_{\theta}(f)(f$ is here a dummy variable) discretized as a centered finite difference $\left[f\left(\theta_{k+1}\right)-f\left(\theta_{k-1}\right)\right] / 2 \delta$, the operator $\partial_{\theta}^{2}(f)$ by $\left[f\left(\theta_{k+1}\right)+f\left(\theta_{k-1}\right)-2 f\left(\theta_{k}\right)\right] / \delta^{2}$, and the integral term $\int_{0}^{2 \pi} f\left(\theta^{\prime}\right) d \theta^{\prime}$ by $\sum_{k=0}^{N_{\text {grid }}-1} f\left(\theta_{k}\right) \delta$, with $\delta=2 \pi / N_{\text {grid }}$.

The two-parameter bifurcation diagram of this equation as a function of the coupling strength and noise are depicted in Fig. 8(b). The bifurcation diagram is organized around two codimension-two Bogdanov-Takens bifurcations, sharing the same Hopf, homoclinic, and saddle-node bifurcation manifolds (two cusp bifurcations were also found and have no impact on the noise-induced oscillations phenomenon). We recover, as in the FitzHugh-Nagumo or the Morris-Lecar system, an eye-shaped region of noiseinduced oscillations for intermediate values of noise and coupling, splitting the parameter space between clamping and asynchronous regimes.

We emphasize that the possibility to access the bifurcation diagram for the probability distribution of the mean-field Fokker-Planck equation allows supporting the conjecture related to the type of bifurcations surrounding the noise-induced oscillations regime. We indeed find that the homoclinic bifurcation arises for lower values of noise than the Hopf bifurcation, consistently, for all coupling strengths. For a fixed value of coupling allowing noise-induced oscillations, the oscillations will emerge from the clamped regime, as noise is increased, through the homoclinic bifurcation, and disappear through a Hopf bifurcation leading to the asynchrony regime.

\section{Noise-induced oscillations in a two-populations Wilson-Cowan equation}

We conclude our analysis of universality with the study of a firing-rate model shown to exhibit noise-induced oscillations [28], and for which one can rigorously access the bifurcation diagram for the mean-field solutions. This model describes the activity of an excitatory $(E)$ and an inhibitory $(I)$ neuron population of size $n_{e}$ and $n_{i}$ :

$$
\begin{aligned}
& d x_{t}^{i}=\left(-x^{i}+\frac{J_{e e}}{n_{e}} \sum_{j=1}^{n_{e}} S\left(x^{j}\right)+\frac{J_{e i}}{n_{i}} \sum_{j=1}^{n_{i}} S\left(y^{j}\right)+I_{e}\right) d t+\sigma d W_{t}^{i}, \\
& d y_{t}^{i}=\left(-y^{i}+\frac{J_{i e}}{n_{e}} \sum_{j=1}^{n_{e}} S\left(x^{j}\right)+\frac{J_{i i}}{n_{i}} \sum_{j=1}^{n_{i}} S\left(y^{j}\right)+I_{i}\right) d t+\sigma d \tilde{W}_{t}^{i},
\end{aligned}
$$

where $I_{e}\left(I_{i}\right)$ is the deterministic level of current received by excitatory (inhibitory) cells and $W^{i}\left(\tilde{W}^{i}\right)$ are independent Brownian motions accounting for current fluctuations. Cells are coupled through the product of a nonlinear sigmoidal (smooth) transform of each neuron's activity multiplied by a coupling coefficient $J_{\alpha \beta}$ for $\alpha, \beta \in\{e, i\}$ representing the typical coupling strength of neurons of population $\beta$ onto neurons of population $\alpha$; these coefficients are assumed to be equal to $J_{\alpha \beta}=J g_{\alpha \beta}$ for a fixed connectivity matrix $G=\left(g_{\alpha \beta}\right)_{\alpha \beta}$ and where $J$ acts as a scaling coefficient as in the previous cases. As shown in Ref. [28], this system converges to the mean-field equations:

$$
\begin{aligned}
& d x_{t}=\left\{-x_{t}+J_{e e} \mathbb{E}\left[S\left(x_{t}\right)\right]+J_{e i} \mathbb{E}\left[S\left(y_{t}\right)\right]\right\} d t+\sigma d W_{t}, \\
& d y_{t}=\left\{-y_{t}+J_{i e} \mathbb{E}\left[S\left(x_{t}\right)\right]+J_{i i} \mathbb{E}\left[S\left(y_{t}\right)\right]\right\} d t+\sigma d \tilde{W}_{t},
\end{aligned}
$$

which are implicit stochastic differential equations with dynamics coupled to the mean of the solution. These are generally complex mathematical equations to handle, but this particular case enjoys a massive simplification. Indeed, the coupling terms in this limit are deterministic expectations, and thus because of the Gaussian nature of the noise and linearity of the intrinsic dynamics, solutions to these stochastic equations are asymptotically Gaussian (or Gaussian for all times if the initial condition is), and its moments satisfy the ODE:

$$
\begin{aligned}
\dot{\mu}_{e} & =-\mu_{e}+J_{e e} f\left(\mu_{e}, v\right)+J_{e i} f\left(\mu_{i}, v\right), \\
\dot{\mu}_{i} & =-\mu_{i}+J_{i e} f\left(\mu_{e}, v\right)+J_{i i} f\left(\mu_{i}, v\right), \\
\dot{v} & =-2 v+\sigma^{2},
\end{aligned}
$$

with $f(\mu, v)=\int_{\mathbb{R}} S(x) e^{-(x-\mu)^{2} / 2 v} / \sqrt{2 \pi v} d x$. This function has the closed form $f(\mu, v)=\operatorname{erf}\left(g \mu+\theta / \sqrt{1+g^{2} v}\right)$ when $S(x)=\operatorname{erf}(g x+\theta)$ with erf the error function (the repartition function of the Gaussian (see Appendix A of Ref. [28]). This simplification thus allows addressing the existence of transitions in the collective dynamics using classical bifurcation theory for ODEs.

This system is not expressed as an excitable system and does not have an explicit slow-fast structure. However, the geometry of its phase plane endows it with excitable properties. Indeed, a single pair of excitatory-inhibitory neurons in the absence of noise features a single stable fixed point, a saddle, and an unstable spiral [see Fig. 8(c1)]. The unstable manifold of the saddle describes a large loop around the unstable fixed point (a heteroclinic orbit), and its stable manifold acts as an excitability threshold: perturbations of the fixed point across this manifold lead to a long excursion back to the stable fixed point along the unstable manifold heteroclinic orbit. We thus expect to find a similar transition to synchrony due to noise, provided that connections are synchronizing. Here, the excitatory-inhibitory nature of the system plays this role and maintains a cohesion in the system (although more indirectly than the electrical coupling). Indeed, when many excitatory neurons are activated, they will activate more excitatory cells, and when a majority is silent, other neurons tend to remain silent: therefore, excitatory neurons may act as the excitable voltage in the FitzHugh-Nagumo or Morris-Lecar model, and inhibition may act as the recovery variable. 
The mechanisms described in this paper thus account for the noise-induced oscillations observed in Ref. [28]: for small noise, the system thus remains in the vicinity of the fixed point, but as noise increases, excursions through the stable manifold of the saddle will be more frequent, raising the number of excited neurons, and eventually leading to a collective spike. Transitions as a function of noise and a global connectivity parameter $J$ scaling all connectivity coefficients are provided in Fig. 8(c2), and confirm again the conjecture of the appearance of noise-induced oscillations through a homoclinic bifurcation at $\sigma$ small and their disappearance through a Hopf bifurcation for $\sigma$ large.

\section{Beyond identical cells and all-to-all coupling}

Realistic networks in the brain are heterogeneous. Cells may differ in the way they process the input, and show variable connectivity patterns, generally sparse. Ample evidence indicates variability in cell properties [93-96] and brain connectivity (see, e.g., Ref. [97], and references therein). Much theoretical work has also shown how variability in cell properties or network architectures impact collective dynamics and synchronization (see Ref. [98] for a review). The model we studied throughout the paper was composed of identical cells coupled to all other cells with the same amplitude, yet the mechanisms we uncovered in this simplified model are more general. To outline the universality of these observations beyond identical cells and all-to-all coupling, we present multiple situations in which the mechanisms persist and discuss some settings where the proposed mechanisms break down.

\section{Randomly connected networks}

Mean-field theory ensures that the results reported for our network persist when the connectivity coefficient between neurons $i$ and $j$, denoted $J_{i j}$, are independent random variables with finite mean and variance. The generalized network equations now read:

$$
\begin{aligned}
d v_{t}^{i} & =\left[f\left(v_{t}^{i}\right)-w_{t}^{i}+\frac{1}{n} \sum_{j=1}^{n} J_{i j}\left(v_{t}^{j}-v_{t}^{i}\right)\right] d t+\sigma d W_{t}^{i}, \\
d w_{t}^{i} & =\varepsilon\left(b v_{t}^{i}-w_{t}^{i}\right) d t,
\end{aligned}
$$

Theoretically, the asymptotic dynamics of this system is identical to that of system (1) with $J$ the average value of the $J_{i j}$. Let us for instance discuss the biologically realistic case of sparsely connected networks. Instead of the case where neurons communicate with all other neurons, we assume that the probability for a synapse to exist between any two neurons is fixed equal to $p$. For simplicity, we assume that when a connection is present, the synaptic weight is deterministic and equal to $J / p$ (random synaptic weights amplitudes with mean $J / p$ should provide the same result). In this setting, $J_{i j}$ are independent random variables equal to $J / p$ with probability $p$ and to 0 otherwise. Figure 9(a) represents the dynamics of this (a) Sparsely connected network

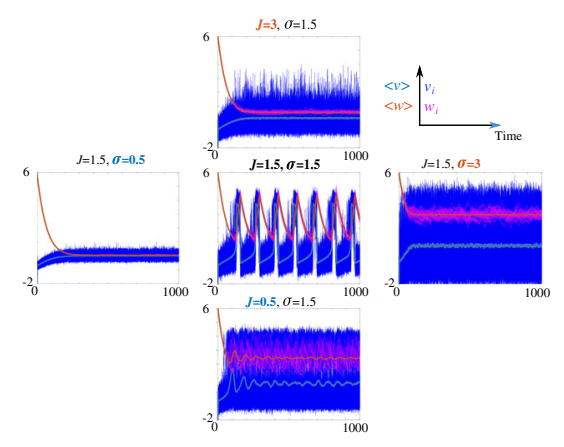

(b) Ring network

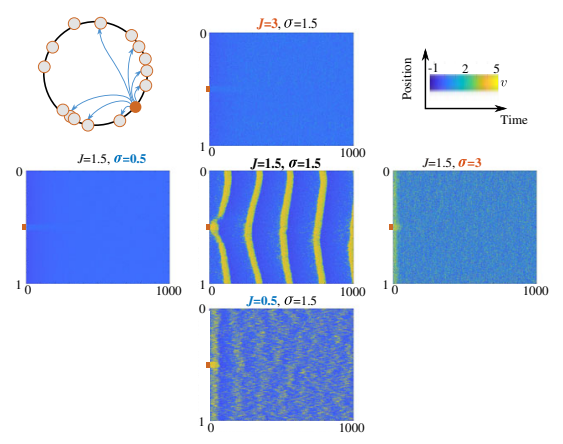

(c) Heterogeneous network

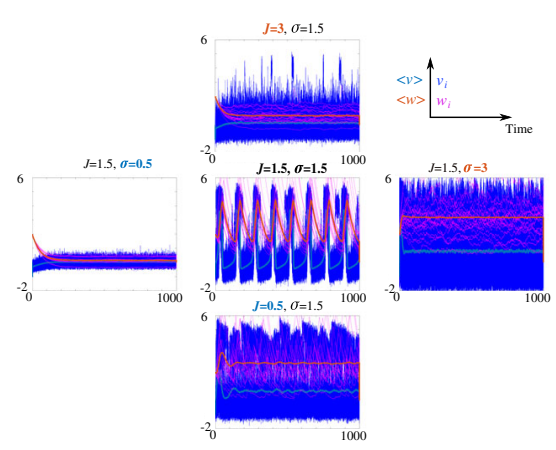

FIG. 9. Noise-induced synchronization in heterogeneous FitzHugh-Nagumo networks model, with $n=4000$ neurons $[n=2000$ for spatial networks in (b)], $a=4, b=4, \varepsilon=0.01$, and $I=0$, for various values of the coupling strength $J$ and noise level $\sigma$. (a) Sparsely connected networks with probability of connection $p=0.6$, and connectivity amplitude $J / p$. Same color code as in Fig. 1 . The graphs represent 10 randomly chosen trajectories of voltage and recovery variable as well as their statistical mean. The system shows an almost identical phenomenology as in the homogeneous network. (b) Spatially extended network with local exponential coupling, i.e., with a probability of connection between two neurons at a distance $D$ given by $e^{-D / \delta}$ with $\delta=0.02$ (see upper left inset for a sketch of the local coupling) and a weight of connection equal to $J / \bar{p}$, where $\bar{p}$ is the average connectivity level of a given neuron. Initial condition was set up with an average excited region of $5 \%$ of the network (100 cells upon 2000$)$. We observe that the phenomenology persists and that a local theory of pioneers and resting neurons seems at play in the emergence of patterns of synchrony for $J=1.5$ and $\sigma=1.5$. (c) Heterogeneous elements. Neurons have heterogeneous excitability levels $a_{i}=a+\sigma_{a} \xi_{t}^{a, i}$ and heterogeneous inputs $I^{i}=I+\sigma_{I} \xi_{t}^{I, i}$, where $\xi^{x, i}$ for $x=a, I$ are white noise, i.e., independent and identically distributed normal centered variables, $\sigma_{a}=0.5$ and $\sigma_{I}=1$. While the dispersion of neurons voltage and recovery variable seems now broader, the phenomena are exactly recovered. 
network for $n=2000$ neurons in each of the five special cases studied throughout the paper, and confirms that this sparsely connected network behaves exactly as the homogeneous case and shows identical transitions.

The emergence of oscillations can be accounted for in the same framework. First, we can readily extend the definition of pioneers and resting neurons to this case, and when $n$ is large, the critical fraction of pioneers can be evaluated using Eq. (2), precisely as in the homogeneous case. Indeed, since the interaction term used in these equations is an averaged force, then on average, the sparsely connected case induces identical forces as in the homogeneous case. Moreover, and for the same reasons, transitions from rest to pioneer and pioneer to rest occur at rates identical to the homogeneous all-to-all network. Therefore, sparsely connected networks (with the proper renormalization of the connectivity weight) display the same phenomenology as homogeneous networks. For small noise or large average coupling coefficient $J$, transitions from the resting state will be rare, because of the rarity of transitions from rest to pioneer preventing their accumulation, and for coupling too low or noise too high, asynchronous dynamics shall arise, while for appropriate noise and coupling, in the exact same range as evaluated for the homogeneous case, the network will oscillate in synchrony.

Other cases of random connectivity for which identical phenomenology arises are cases of independent random connections $J_{i j}$ with mean connection $J$ and a variance growing slower than $n^{1-\kappa}$ for some $\kappa \in(0,1)$, as in all these cases the fluctuations associated with random connections will vanish in the limit. This picture breaks down, however, when randomness becomes too strong, for instance, for synaptic weights with variance proportional to the network size. In that case, the fluctuations associated with variable synaptic weights become prominent and can change the phenomenology, as was observed, for instance, in the celebrated work of Sompolinsky et al. [99]. We for instance showed, both in the case of the electrically coupled FitzHugh-Nagumo equations as well as in a WilsonCowan network similar to the one studied here, that when the variance of the synaptic coefficient is proportional to the network size, transitions due to the variance of the synaptic weight do occur [100]. We note that the phenomena leading to the synchronization due to heterogeneous connections in these systems likely do not rely on similar phenomena, and it remains an open question to characterize the microscopic phenomena at play leading to heterogeneity-induced oscillations. Besides networks with connectivity having diverging variances, cases that are not covered by the theory are ultrasparse networks where the probability of connection $p_{n}$ go to 0 as $n \rightarrow \infty$, such as networks where neurons can communicate only with a finite number of other cells. In that case, it would indeed not be possible to maintain a nontrivial level of connectivity (as we did when $p_{n}$ was constant by rescaling the coefficient), and other scalings may be used.

\section{Spatial networks and excitable media}

Although connectivity was nonhomogeneous in the network considered in Sec. VC1, these networks are composed of exchangeable elements, in the sense that the coefficients $J_{i j}$ were considered independent and identically distributed, and all elements had identical dynamics. This type of connectivity neglects spatial effects. In many applications, excitable elements are organized spatially, forming excitable media. This is the case of nerve cables (axons), a piece of cortex, or the BelousovZhabotinsky reaction. An abundant literature deals with the dynamics of excitable media and the impact of noise on these systems [7,101]. It is well known that such media can support global oscillations and traveling waves, in particular. We expect that similar phenomena may be at play in the emergence of such patterns in response to noise, and that these could rely on similar microscopic mechanisms as described in this paper. In detail, a theory of how microscopic phenomena support the emergence of traveling waves or global oscillations could indeed be developed along the lines of the present work, but relying on a local notion for the fraction of pioneers. We illustrate this phenomenon in Fig. 9(b) on a ring network, where each neuron has a random location on the circle $\mathbb{S}^{1}$ and randomly connects to other neurons with a probability decreasing with the distance. For definiteness, we chose a coupling probability decaying as an exponential according to the distance between neurons. In detail, the probability for neuron $i$ at location $r_{i}$ to communicate with neuron $j$ at location $r_{j}$ is given by $J_{i j}=J e^{-\left\|r_{i}-r_{k}\right\| / \delta}$, where $\delta$ is the typical connectivity distance. We set up the initial condition near the rest state, except for a small region of the medium for which the system is composed of pioneers (initial condition in the excitable region). Locally, these pioneers will have the ability to excite other cells at the appropriate amount of noise and connectivity, much like a local homogeneous network, thus propagating a wave of excitation for such parameters, followed by a return to rest and then a progressive return to the excited regime. Figure 9(b) indeed highlights this phenomenon and recovers noiseinduced pattern formation in this system, with a structured propagation of the excited initial state only for specific values of noise and coupling, and a consistent evolution of local pioneers. Beyond showing this numerical evidence, the development of a detailed microscopic theory of pattern formation in excitable media is beyond the scope of the present paper.

\section{Heterogeneous elements}

We close our discussion on the universality of the mechanisms by mentioning how the theory may extend to networks composed of heterogeneous excitable elements. To this end, we considered a network equation with neurons of heterogeneous excitability. In detail, we considered the system 


$$
\begin{aligned}
d v_{t}^{i}= & {\left[v_{t}^{i}\left(1-v_{t}^{i}\right)\left(v_{t}^{i}-a^{i}\right)-w_{t}^{i}+\frac{1}{n} \sum_{j=1}^{n} J_{i j}\left(v_{t}^{j}-v_{t}^{i}\right)+I^{i}\right] d t } \\
& +\sigma d W_{t}^{i}, \\
d w_{t}^{i}= & \varepsilon\left(b v_{t}^{i}-w_{t}^{i}\right) d t
\end{aligned}
$$

where $a^{i}$ and $I^{i}$ are no more constant but fluctuate across neurons. For definiteness we chose these parameters to be independent Gaussian random variables centered at the values of reference $a=4$ and $I=0$ used throughout the paper, and with standard deviation 0.5 and 1 [102]. Both parameters control the excitability of the neurons, and thus while the averaged excitability is similar to the homogeneous network, the network Eq. (11) is composed of elements with varying excitability levels. While we expect that a theory based on following fractions of pioneers and determining critical fractions leading to a chain reaction shall allow accounting for these dynamics, such a theory would need specific care in defining pioneers and transition rates. Indeed, in such a heterogeneous system, neurons will transition from rest to pioneer and pioneer to rest at distinct rates, as the most excitable neurons can be driven to the pioneer state in response to smaller input. Similarly, heterogeneity will impact each individual neuron's critical fraction, since for instance less excitable cells will need a higher proportion of pioneers to be drawn to transition to the pioneer state. However, we conjecture that the mechanisms described in this paper should persist statistically. Figure 9(c) shows indeed that such networks closely recover the phenomenology of the homogeneous network, although more fluctuations in the individual trajectories may arise.

\section{DISCUSSION}

Noise is a prominent feature in natural or physical systems, arising from multiple sources including electrical fluctuations, thermal agitation of molecules or electrons, and synaptic activity, to cite a few. In nonlinear systems, noise can have multiple effects, such as attractor switching in multistable systems, stochastic resonances in excitable systems [7], or inverse resonances near folds of limit cycles $[103,104]$. These phenomena have been largely studied for finite-dimensional stochastic systems. The role of noise in large-scale nonlinear interacting particle systems is significantly less well understood. A variety of articles have revealed the complex phenomenology noise may have in these systems, and particularly, a regularizing effect leading to the stabilization of stationary solutions or the emergence of periodic solutions [7,26,27,29,30,33]. Despite increasing evidence and abstract mathematical proofs in the meanfield limit regime, the origins of these surprising regularizing effects of noise have remained elusive.

In the first part of this paper, we thoroughly investigated this collective phenomenon of robust emergence of synchronized oscillations. Two essential microscopic mechanisms were highlighted underlying this behavior: asymmetric transitions from rest to an excited state and vice versa, leading to an increase in the fraction of excited neurons, and a subsequent chain reaction recruiting all neurons into a collective excursion. Contrasting with other classical meanfield behaviors, these dynamics are therefore not driven by an influence of the ensemble average itself, but rather by the random and independent fluctuations of each neuron's activity, enabling the buildup of a macroscopic fraction of neurons in the excited state. In a sense, and contrasting with stochastic resonance for single elements, coupling and noise conspire here to yield a collective resonance phenomenon, which ends up yielding exactly periodic responses. These phenomena are not specific to the FitzHugh-Nagumo model, but are universal to stochastic networks of excitable elements with confining interactions, as we confirmed in three other classical neural networks.

The emergence of oscillations at unison arising in response to independent stochastic fluctuations of each element may seem surprising: one may have expected stronger correlations in the average activity in response to correlated inputs, or small noise. It is however an opposite effect that takes over: regular macroscopic trajectories emerge actually in response to having independent noise, making each neuron independent in the large network size limit, and thus the time to reach the critical fraction of excited neurons deterministic, much like an empirical average of independent variables, converges to a deterministic value in the law of large numbers. Independent noise at the level of each neuron is a relatively realistic assumption in the brain when accounting for the ongoing bombardment of synaptic inputs and channel fluctuations, and was supported by recent data $[105,106]$ and general properties of large-scale stochastic networks.

This phenomenon shares a number of commonalities with brain activity disruptions occurring in Parkinson's disease. Indeed, in this disease, abnormal oscillations emerge and are associated with an increased excitability of cells $[47,78]$ and enhanced electrical transmission [79]. Motivated by this analogy, we next investigated the possible impact of high-frequency stimulation on the network, emulating DBS therapies. We observed that such a periodic forcing could prevent the emergence of synchrony. For this desynchronization to occur, stimulation shall have high frequency relative to the frequency of intrinsic oscillations, but not too high. Low-frequency stimulations forced the system to lock to the stimulus, very high frequency stimulations had no impact on the spontaneous oscillations. Between these two regimes, the system, poised near the chain reaction threshold, maximized the correlation between the input and output and other measures of information transmission (e.g., mutual information).

Undoubtedly, our model does not constitute a realistic neural population architecture and connectivity appropriate 
to reproduce closely the phenomena arising in Parkinson's disease. Despite this simplification, several parallels can be drawn between our model and clinical observations of parkinsonian patients $[54,57,80,107]$. In particular, the fact that DBS efficiency depends on the frequency of stimulation and that optimal outcomes on motor symptoms and on the abolishment of beta oscillations $(10-30 \mathrm{~Hz})$ arise for high-frequency stimulations (typically, around $130 \mathrm{~Hz}$ ); the ratio between this effective stimulation frequency and the frequency of the intrinsic oscillatory rhythm is similar to what we observe in our model, both for the emergence of the desynchronized regime and maximal information transmission capability (Pearson's coefficients or mutual information). Moreover, the amplitude of stimulation pulses can be optimized: our model indicates that a robust increase in information transmission capabilities can be found for relatively low-amplitude stimulation, as soon as the desynchronization occurs. Therapeutically, low-amplitude stimulation could seem beneficial since it avoids imposing high-amplitude currents that may be detrimental to network activity and may prevent the network from responding with a high signal-to-noise ratio to natural stimuli. In our simple model with a single electrically coupled neuronal population, the mechanisms by which periodic forcing can abolish oscillations relies on a sequence of excitations compensated by inhibition, and is thus valid only for biphasic stimulations such as the Lilly pulse. In the FitzHugh-Nagumo model, monophasic excitatory input would not interrupt oscillations, as excitation alone will only hasten macroscopic spikes. In constrast, a monophasic inhibitory input would abolish oscillations, but this effect would be monotonic in frequency, and therefore would not recover the two opposite effects observed for too-low or too-high frequencies both in the model under biphasic stimulation and clinically. The model, however, does not suggest that biphasic pulses should be more beneficial clinically than monophasic pulses. Testing this hypothesis would require studying a significantly more realistic model including other types of interactions distinct from electrical coupling, and multiple neuronal populations, in particular inhibitory cells that may play a crucial role in desynchronization; see, e.g., Ref. [108]. This stresses the importance of studying the dynamics of more realistic networks, made of multiple populations and including the presence of chemical-like pulse coupling, in particular under the various frameworks available in the literature $[109,110]$.

In contrast to more realistic models of cortico-basal ganglia loops aiming at explaining the detailed mechanisms underlying the emergence of oscillations and the therapeutic effect of DBS [59,111-113], we propose a more abstract line of thought linking macroscopic level to microscopic dynamics. In particular, our model proposes an alternative view to the "information lesion" hypothesis [114], suggesting that instead of producing highly regular output patterns, DBS endows the network with highly variable spontaneous activity, without any strong coherent activation of individual neurons, and thus high information transmission. The type of loss of synchrony evidenced here is quite distinct from the mechanisms suggested in more realistic models. In particular, the work of Rubin and Terman [59] relies on detailed modeling of the interaction between the multiple populations composing the basal ganglia. This model specifically allows studying how DBS modifies the patterns of spikes of inhibitory populations (particularly, the internal segment of the globus palidus), whose spiking pattern turns from rhythmic bursting to a stronger, but tonic firing. Moreover, the effect being present only in a given window of frequency distinguishes our model from those relying on patterns of increased inhibition, blockade, or stochastic phase resetting [112]. It is also distinct from the stochastic phase resetting theory elegantly proposed by Tass [115] extending Winfree's work on oscillators responses to pulses [116] to populations of oscillators, in that our mechanism does not rely on neurons being oscillators but, instead, excitable elements synchronizing in response to noise. We further emphasize that the stochastic phase resetting theory opened the way to a current line of theoretical and clinical research on adaptive DBS stimulation protocols, either in closed-loop settings responding to brain's activity $[112,113,117-119]$ or aiming at providing the stimulation on demand, mostly in the context of obsessive-compulsive disorder treatment [120]. Our study suggests that accumulation of pioneers is an early indicator of collective spikes and could be used to refine electrical stimulations in a closed-loop system in a similar manner as proposed in these detailed modeling and clinical works. Moreover, the arguments we provided accounting for the loss of synchronization are, again, not specific to the particular model studied, and we argue that this phenomenon should arise also in more realistic situations. In particular, the same type of protocols and background was used in a more realistic model of the cortico-striatal loop in normal and parkinsonian conditions [108], and this work opens up a promising avenue for a better understanding of Parkinson's disease and its treatment. The study also highlights a number of open problems in mathematics: most phenomena described indeed challenge a well-developed theory of noise in nonlinear dynamics and call for extending these results beyond small noise and single elements.

\section{ACKNOWLEDGMENTS}

The authors warmly thank Bertrand Degos and Marie Vandecasteele for multiple discussions on Parkinson's disease, as well as Valentin Figué for his research work in the early stages of the study. Partial funding was provided by the Fondation Bettencourt Schueller. 


\section{APPENDIX A: TRANSITION RATES AND KRAMERS' THEORY}

In the main text, we derived numerically the transition rates from pioneer to rest and reciprocally through numerical simulations. These rates are analogous to transition rates of stochastic particles in multiwell potentials, and we discuss here the limitations of using the well-developed classical theory of attractor switching in multiwells potentials to the problem at hand.

For a stochastic particle in a multiwell potential and in the limit of small noise, it is well known that the particle switches attractor at exponentially distributed times with rates depending on the depth of the wells and the noise level $[71,72]$. Here, the problem at hand challenges the standard theory in many ways. One important difficulty stems from the fact that noise-induced oscillations arise for nonvanishing noise, a regime where little remains known about transitions between multiple attractors and where largedeviations estimates are ineffective. Moreover, each particle has an excitable dynamics instead of a multistable Hamiltonian dynamics: while rest is indeed an equilibrium, the pioneer state is a transient passage that does not correspond to a stable fixed point.

One way around the latter difficulty is to reduce the system to a one-dimensional equation with $w$ a constant during the time of a given transition, as done multiple times in Sec. II. This allows deriving an effective potential to approximate system during the transition phase. Unfortunately, the potential obtained would now depend on the level of noise and of the distribution of the voltages of neurons. Indeed, Eq. (4) corresponds to the dynamics of a noisy particle in a one-dimensional potential $U$ given by

$$
\begin{aligned}
U(v) & =-\int_{0}^{v} f(y) d y+J \frac{v^{2}}{2}+A v \\
& =\frac{v^{4}}{4}-\frac{(1+a) v^{3}}{3}+\frac{(J+a) v^{2}}{2}+A v
\end{aligned}
$$

with $A=w_{0}-J(1-\alpha) v_{r}+\alpha v_{p}$. This potential requires assuming that the system, during one transition, conserves a fixed value of $w_{0}$, a fixed fraction $\alpha$ of neurons in the vicinity of a pioneer state $v_{p}$ and the rest of neurons in the vicinity of the resting potential $v_{r}$. Both assumptions are reasonable for the system at hand, but two difficulties arise to obtain analytical results using this formula. First, the potential depends on noise and coupling through $w_{0}$. Moreover, $v_{r}$ and $v_{p}$ are also not fully defined and depend on $w_{0}$ (thus on noise and $J$ ) and $\alpha$. Indeed, $v_{r}$ and $v_{p}$ shall correspond to the resting and pioneer state, two putative minima of the potential. Computing these quantities thus amounts to solving an implicit equation:

$$
f(v)-J v+w_{0}-J\left[(1-\alpha) v_{r}+\alpha v_{p}\right]=0,
$$

where $v_{r}$ is the smallest and $v_{p}$ is the largest actually solution of the cubic equation, when that equation has three solutions. These in particular only exist for a limited range of values of $(\alpha, \sigma, J)$ for which $U$ is a double-well potential. The dependence in $w_{0}$ of the potential highlights the interplay between noise and nonlinearity in the system, while the dependence in $\alpha$ shows the collective nature of the problem, both being fundamental differences with the classical attractor switching framework.

Despite these differences, we computed, when possible, the effective potential of the system. To this purpose, we derived the values of $v_{r}, v_{p}$ using a fixed-point method. In detail, fixing $v_{r}^{0}$ and $v_{p}^{0}$ as the smallest and largest solutions of $f(v)=w_{0}$, we iteratively solved (when possible) the equation

$$
f\left(v^{n+1}\right)-J v^{n+1}+w_{0}-J\left[(1-\alpha) v_{r}^{n}+\alpha v_{p}^{n}\right]=0
$$

for $n \geq 0$ and checked for convergence of the sequence $\left(v_{r}^{n}, v_{p}^{n}\right)$. Convergence of that sequence ensured the fact that the effective potential has indeed a double-well profile. Denoting $v_{u}$ the voltage associated with the saddle (unstable fixed point), the Kramers-Freidlin-Wentzell theory of attractor switching provides an asymptotic expansion of the escape time depending on the shape of the potential $U$ when $\sigma$ is small:

$$
\frac{2 \pi}{\sqrt{-U^{\prime \prime}\left(v_{u}\right) U^{\prime \prime}\left(v^{*}\right)}} \exp \left(\frac{U\left(v_{u}\right)-U\left(v^{*}\right)}{\sigma}\right),
$$

where $v^{*}$ is the voltage of one of the stable nodes of the system $\left(v_{p}\right.$ or $\left.v_{r}\right)$.

Figure 10 compares the rates provided by the KramersFreidlin-Wentzell theory with those obtained numerically in Sec. III B. We found an excellent agreement of the rates where it can be computed (presence of a double-well potential) and when the wells are deep enough. In detail, rest and pioneer states for the potential can be defined for a finite interval of values $\alpha$; for $\alpha$ too small, the pioneer state is barely attractive to a particle starting from the resting regime, and for $\alpha$ too large, the resting state becomes progressively less stable and even disappears. In flat regions of the potential, the analytical rate deviates from the numerical rate computed and overestimates the time it will take for a particle to cross the quasiseparatrix. Indeed, a noisy particle in a potential whose depth is of the same order of magnitude as typical amplitude of stochastic excursions will have a larger transition rate than predicted by the theoretical asymptotic escape rate in small noise. The numerical method used in Sec. III B avoids these difficulties and computes the effective transition times regardless of the assumptions needed for applying the Kramers-Freidlin-Wentzell theory. 


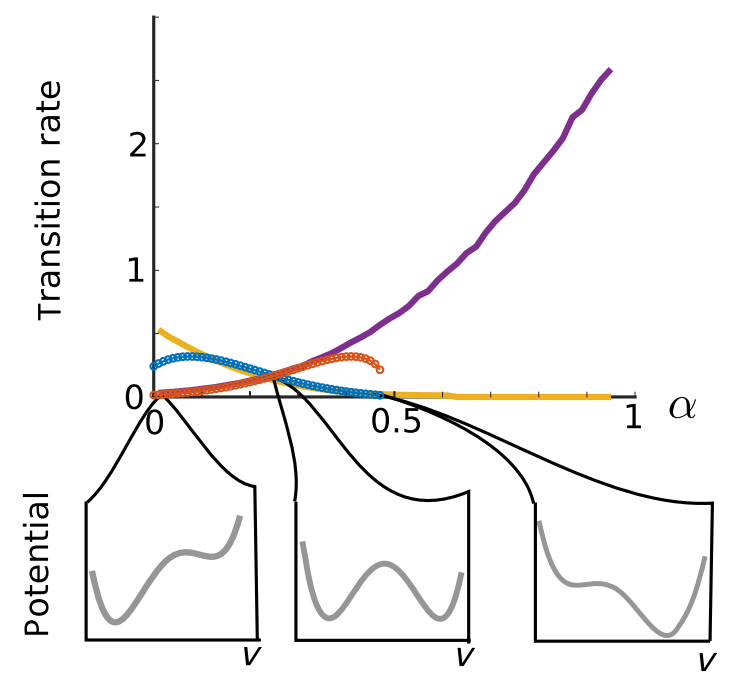

FIG. 10. Comparison of numerical rates of transition from pioneer to rest (yellow) or rest to pioneer (purple) with associated Kramers escape rate formula (A1) (respectively, blue and red) for $J=1.5$ and $\sigma=1.5$. A very good agreement arises when the equilibria are sufficiently stable (potential wells of $U$ deep enough). Escape rates are largely underestimated when the potential becomes too flat (conjectured breakdown arising when the depth becomes of the same order of magnitude as the potential well depth), in which case typical stochastic fluctuation dominates the escape rate compared to the small-noise correction provided by the theory.

\section{APPENDIX B: MUTUAL INFORMATION MAXIMIZATION}

In the main text, we showed that information transmission is enhanced in the regime where a periodic stimulus abolishes noise-induced oscillations. These results were based on Pearson's correlation coefficients between the average voltage and an input signal. Here, we confirm these results using an alternative methodology relying on computing mutual information. While both correlations and mutual information measure distances from independence between two random variables, correlations are limited to evaluate linear relationships between random variables while mutual information provides refined information about the joint distribution of two random variables. Technically, information is evaluated through the entropy of the responses. Here, because of the emergence of periodic responses for too slow or too fast DBS stimulation, direct estimations of the entropy over the full trajectory will underestimate the actual information content. To overcome this limitation, we calculated how input modified response patterns within a time window of size $\Delta t$ evaluated according to the spontaneous period of the network responses in the absence of stimulus (period evaluated through the Fourier transform of the solutions). Computing entropies requires constructing the histogram of the responses, which, for a given stimulus, shall require many repetitions of the stimulus. Here, the propagation of chaos property (demonstrated in Ref. [65]) ensures that, in the limit $n \rightarrow \infty$, the neurons are independent realizations of the same mean-field process; we used this property to approximate the distribution of the voltage of a given cell by the histogram distribution of the voltage of all neurons. Moreover, we combined data from 100 repetitions of the same network for each stimulus considered.

We thus computed a stimulus-specific windowed entropy $H_{s}$ as an averaged entropy across time windows $[k \Delta t,(k+1) \Delta t)$ by computing the probability distribution of the voltage or recovery variables over all neurons within a given window in response to a given stimulus. In detail, we numerically computed the solution of the stochastic FitzHugh-Nagumo equation $\left[v^{i}(t), w^{i}(t)\right]$ for $t \in[0, K \Delta t]$, with $K \in \mathbb{N} \backslash\{0\}$ and $i \in\{1, \ldots, n\}$. Given a voltage or recovery resolution $\Delta B_{v, w}$ (chosen to be 0.025 in our simulation), we define fixed partitions of the voltage and recovery variables $\left\{v_{1}, \ldots, v_{M_{1}}\right\}$ (voltage partition) and $\left\{w_{1}, \ldots, w_{M_{2}}\right\}$, and computed a discretized empirical probability distribution $\hat{p}_{v, w}^{\alpha}(k)$ as the probability to find a neuron in the network with a voltage (or recovery variable) within the segment $\left[v_{\alpha}, v_{\alpha+1}\right]\left(\left[w_{\alpha}, w_{\alpha+1}\right]\right)$ during the time interval $[k \Delta t,(k+1) \Delta t)$, with $\alpha \in\left\{1, \ldots, M_{1}-1\right\}$ and $k \in\{0, \ldots, K-1\}$. The stimulus-specific entropy was computed as the associated Shannon's differential entropy:

$$
H_{s}^{v}=\frac{1}{Z} \sum_{k=0}^{K-1} \sum_{\alpha=1}^{M_{1}-1} \hat{p}_{v}^{\alpha}(k) \log \left(\frac{\hat{p}_{v}^{\alpha}(k)}{\Delta B_{v}}\right)
$$

(a similar expression is used for the recovery variable entropy), where $Z$ is a normalization constant (generally $K M_{1,2} \Delta B_{v, w}$ ) that acts as a simple scaling term with no impact on the qualitative results.

Similarly, the averaged windowed entropy $H_{\text {all }}$ was calculated as the average of the stimulus-specific entropies across all stimuli considered, and the mutual information was then defined for each stimulus as

$$
\eta=H_{\text {all }}-H_{s} .
$$

The stimulus set consisted of 100 stimuli for each of three classes of signals: Gaussian white noise with distinct standard deviations (uniformly distributed in $[0,5]$, stationary Ornstein-Uhlenbeck processes (with timescale uniformly chosen in [0.01, 0.05], average $\mu$ uniform in [0,5], and noise level uniform in $[0,5])$ as well as OrnsteinUhlenbeck centered at a cosine (same parameters for as the Ornstein-Uhlenbeck process, with amplitude of cosine uniformly distributed in $[0,5]$ and frequency uniform in $[0,20])$. Entropies were computed based on the empirical distributions on a fixed grid (step: 0.025 , voltage from -3 to 6 , recovery variable from 0 to 10 ). Simulations confirmed the significant increase in the mutual information, spanning the entire band characteristic of the desynchronized regime (here again, for all stimulus type, the correlation between the presence of spontaneous oscillations and 
(a) Mutual information (MI)

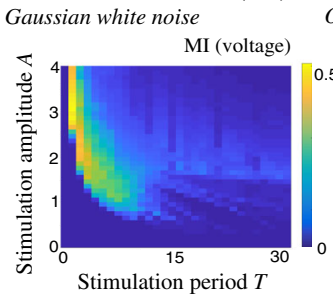

Ornstein-Uhlenbeck
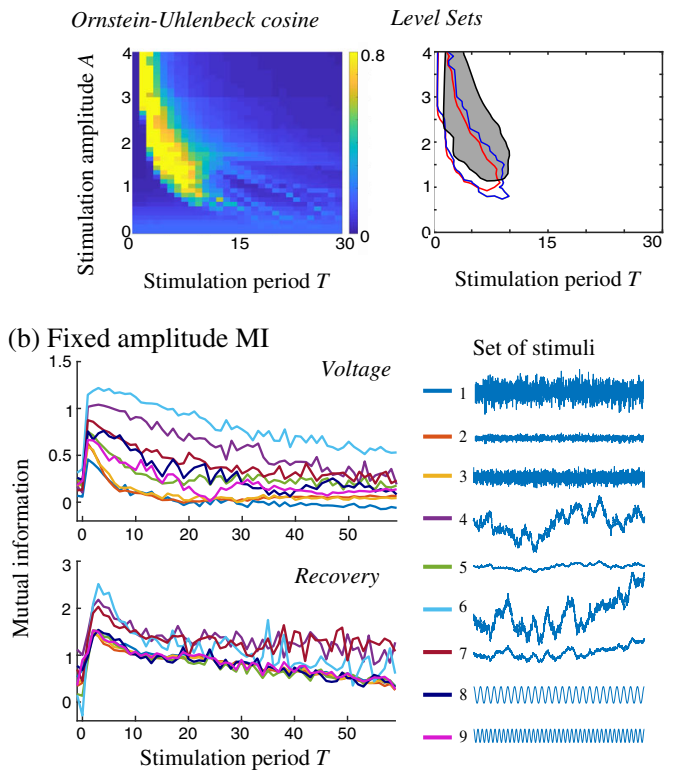

(c) Optimal amplitude
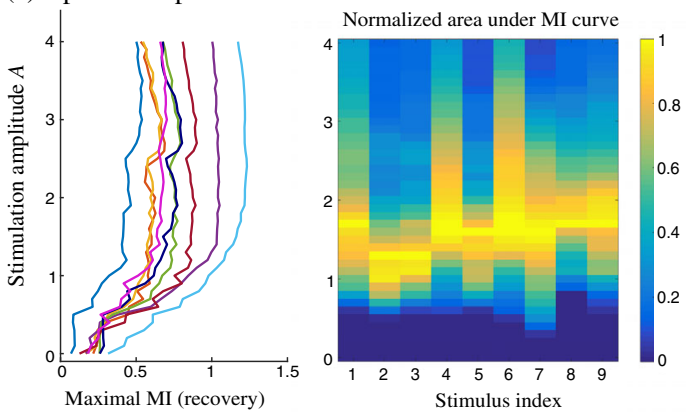

FIG. 11. Mutual information estimation for the FitzhughNagumo network model, parameters and setting as in Fig. 7. (a) Mutual information (recovery variable) as a function of the amplitude and period of the periodic stimulation for Gaussian white noise, Ornstein-Uhlenbeck, or OrnsteinUhlenbeck centered at a sine wave. Lower right-hand panel: Peak of mutual information (identified through a level set in the map) coincides with the disappearance of oscillations (gray region, as in Fig. 7). (b) Mutual information as a function of the period of the periodic stimulus (amplitude $A=2$ ) for 9 sample stimuli highlighting the coincidence of their maxima, both for voltage and recovery variables. (c) The amplitude and width of the peak vary with input amplitude. Left: Maximal value of the mutual information found for each stimulation amplitude: (Right) An optimal amplitude stands out when considering the area under the mutual information curve [normalized by the largest area for each stimulus type highlighted in (b)], suggesting the existence of an optimal stimulation amplitude.

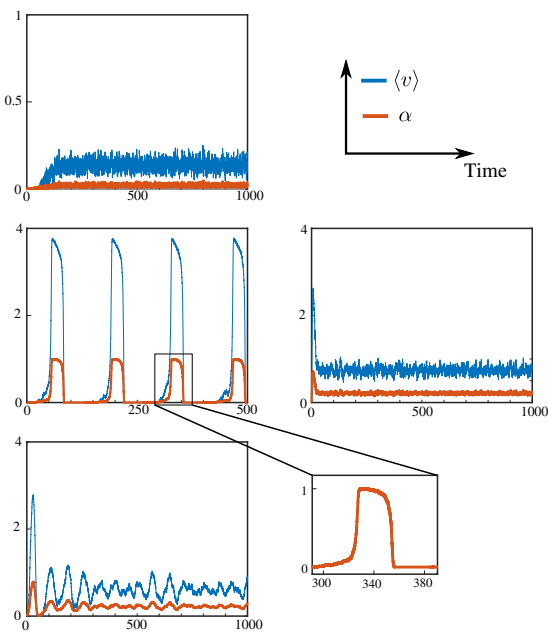

FIG. 12. Dynamics of the fraction of pioneers $\alpha$ as a function of time compared to the average voltage (blue). A smooth and rapid increase of $\alpha$ from 0 to 1 arises in the noise-induced oscillations case at the onset of a spike, while in all other cases $\alpha$ stabilizes.

the mutual information showed strong negative correlations of about $-60 \%$ ), when quantified from both the voltage and recovery variables. This significant elevation of the mutual information in the desynchronized regime can be observed for every stimulus considered, regardless of their nature or their intensity [Fig. 11(c)]. In addition, the mutual information and the amplitude of oscillations are correlated to the stimulation frequency: indeed, the sharp decay of oscillations as the frequency of the periodic forcing is decreased parallels with a sharp rise of the mutual information, while as the regime moves into periodically forced oscillations, the mutual information decreases smoothly. The mutual information between the stimuli and the

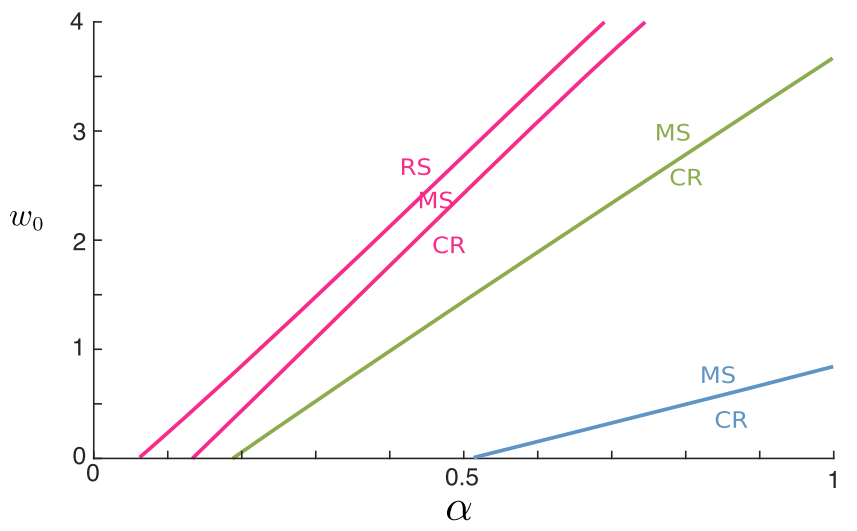

FIG. 13. Two-parameter bifurcation diagram of the simplified system [Eq. (2)] with respect to $\alpha$ and $w_{0}$ for various values of the coupling strength: $J=2.5$ (pink), $J=1.5$ (green), and $J=0.5$ (blue). RS, attraction to the resting state; MS, attraction to mixed states; CR, chain reaction: attraction to the pioneer fixed point. No codimension-two bifurcation is found, indicating no qualitative change in the dynamics as $w_{0}$ is modified (e.g., when noise is varied). 
network responses under low-frequency stimulation also tends to be close to the mutual information characteristic of the intrinsically oscillating regime.

We note that both mutual information and correlation levels vary with the amplitude of the periodic stimulation: indeed, as the amplitude of the periodic stimulation increases, there is an increase in the peak of the mutual information or correlation [Fig. 11(d)], before saturating. Yet, the width of this peak in mutual information has a nonmonotonic relation with the stimulation amplitude: low-amplitude stimulation yields a medium improvement in stimulus encoding over a wide range of frequency, while for high-amplitude stimulation, the increase in mutual information occurs on an increasingly narrow frequency band [as can be seen for two stimuli in Fig. 11(b)]. Therefore, for each stimulus, an optimal stimulation amplitude can be defined, such that the area under the mutual information curve in the desynchronized regime is maximized [Fig. 11(d)]: this optimal amplitude is relatively low, and consistent for all stimuli tested, indicating the presence of an optimal forcing for information transmission regardless of the stimulus type, suggesting the existence of an optimal stimulation frequency for information transmission.

[1] D. Goulding, S. P. Hegarty, O. Rasskazov, S. Melnik, M. Hartnett, G. Greene, J. G. McInerney, D. Rachinskii, and G. Huyet, Excitability in a Quantum Dot Semiconductor Laser with Optical Injection, Phys. Rev. Lett. 98, 153903 (2007).

[2] S. Kadar, J. Wang, and K. Showalter, Noise-Supported Travelling Waves in Sub-Excitable Media, Nature (London) 391, 770 (1998).

[3] J. D. Touboul, A. C. Staver, and S. A. Levin, On the Complex Dynamics of Savanna Landscapes, Proc. Natl. Acad. Sci. U.S.A. 115, E1336 (2018).

[4] L. Glass, Synchronization and Rhythmic Processes in Physiology, Nature (London) 410, 277 (2001).

[5] G. M. Süel, J. Garcia-Ojalvo, L. M. Liberman, and M. B. Elowitz, An Excitable Gene Regulatory Circuit Induces Transient Cellular Differentiation, Nature (London) 440 , 545 (2006).

[6] G. Ermentrout and D. Terman, Mathematical Foundations of Neuroscience (Springer, New York, 2010).

[7] B. Lindner, J. Garcia-Ojalvo, A. Neiman, and L. Schimansky-Geier, Effects of Noise in Excitable Systems, Phys. Rep. 392, 321 (2004).

[8] A. S. Pikovsky and J. Kurths, Coherence Resonance in a Noise-Driven Excitable System, Phys. Rev. Lett. 78, 775 (1997).

[9] B. Lindner and L. Schimansky-Geier, Analytical Approach to the Stochastic FitzHugh-Nagumo System and Coherence Resonance, Phys. Rev. E 60, 7270 (1999).

[10] R. E. Lee DeVille, E. Vanden-Eijnden, and C. B. Muratov, Two Distinct Mechanisms of Coherence in Randomly
Perturbed Dynamical Systems, Phys. Rev. E 72, 031105 (2005).

[11] L. Gammaitoni, P. Hänggi, P. Jung, and F. Marchesoni, Stochastic Resonance, Rev. Mod. Phys. 70, 223 (1998).

[12] J. E. Levin and J. P. Miller, Broadband Neural Encoding in the Cricket Cercal Sensory System Enhanced by Stochastic Resonance, Nature (London) 380, 165 (1996).

[13] J. J. Collins, T. T. Imhoff, and P. Grigg, Noise-Enhanced Information Transmission in Rat SAl Cutaneous Mechanoreceptors via Aperiodic Stochastic Resonance, J. Neurophysiol. 76, 642 (1996).

[14] K. Wiesenfeld and F. Moss, Stochastic Resonance and the Benefits of Noise: From Ice Ages to Crayfish and Squids, Nature (London) 373, 33 (1995).

[15] G. Buzsaki, Rhythms of the Brain (Oxford University Press, New York, 2004).

[16] X.-J. Wang, Neurophysiological and Computational Principles of Cortical Rhythms in Cognition, Physiol. Rev. 90, 1195 (2010).

[17] P. Uhlhaas and W. Singer, Neural Synchrony in Brain Disorders: Relevance for Cognitive Dysfunctions and Pathophysiology, Neuron 52, 155 (2006).

[18] A. Faisal, L. Selen, and D. Wolpert, Noise in the Nervous System, Nat. Rev. Neurosci. 9, 292 (2008).

[19] J. A. Acebròn, L. L. Bonilla, C. J. P. Vicente, F. Ritort, and R. Spigler, The Kuramoto Model: A Simple Paradigm for Synchronization Phenomena, Rev. Mod. Phys. 77, 137 (2005).

[20] S. H. Strogatz, From Kuramoto to Crawford: Exploring the Onset of Synchronization in Populations of Coupled Oscillators, Physica (Amsterdam) 143D, 1 (2000).

[21] B. C. Bag, K. G. Petrosyan, and C.-K. Hu, Influence of Noise on the Synchronization of the Stochastic Kuramoto Model, Phys. Rev. E 76, 056210 (2007).

[22] Y. M. Lai and M. A. Porter, Noise-Induced Synchronization, Desynchronization, and Clustering in Globally Coupled Nonidentical Oscillators, Phys. Rev. E 88, 012905 (2013).

[23] S. Shinomoto and Y. Kuramoto, Phase Transitions in Active Rotator Systems, Prog. Theor. Phys. 75, 1105 (1986).

[24] H. Sakaguchi, S. Shinomoto, and Y. Kuramoto, Phase Transitions and Their Bifurcation Analysis in a Large Population of Active Rotators with Mean-Field Coupling, Prog. Theor. Phys. 79, 600 (1988).

[25] J. Pham, K. Pakdaman, and J. F. Vibert, Noise-Induced Coherent Oscillations in Randomly Connected Neural Networks, Phys. Rev. E 58, 3610 (1998).

[26] M. Scheutzow, Noise Can Create Periodic Behavior and Stabilize Nonlinear Diffusions, Stoch. Proc. Appl. 20, 323 (1985).

[27] M. Scheutzow, Some Examples of Nonlinear Diffusion Processes Having a Time-Periodic Law, Ann. Probab. 13, 379 (1985).

[28] J. Touboul, G. Hermann, and O. Faugeras, Noise-Induced Behaviors in Neural Mean Field Dynamics, SIAM J. Appl. Dyn. Syst. 11, 49 (2012).

[29] E. Luçon and C. Poquet, Emergence of Oscillatory Behaviors for Excitable Systems with Noise and 
Mean-Field Interaction, A Slow-Fast Dynamics Approach, Commun. Math. Phys. 373, 907 (2020).

[30] C. Quininao and J. D. Touboul, Clamping and Synchronization in the Strongly Coupled FitzHugh-Nagumo Model, arXiv:1804.06758.

[31] P. Dai Pra, M. Fischer, and D. Regoli, A Curie-Weiss Model with Dissipation, J. Stat. Phys. 152, 37 (2013).

[32] F. Collet and M. Formentin, Effects of Local Fields in a Dissipative Curie-Weiss Model: Bautin Bifurcation and Large Self-Sustained Oscillations, J. Stat. Phys. 176, 478 (2019).

[33] M. Zaks, X. Sailer, L. Schimansky-Geier, and A. Neiman, Noise Induced Complexity: From Subthreshold Oscillations to Spiking in Coupled Excitable Systems, Chaos 15, 026117 (2005).

[34] C. Kurrer and K. Schulten, Noise-Induced Synchronous Neuronal Oscillations, Phys. Rev. E 51, 6213 (1995).

[35] K. Dierkes, F. Jülicher, and B. Lindner, A Mean-Field Approach to Elastically Coupled Hair Bundles, Eur. Phys. J. E 35, 37 (2012).

[36] T. Prager, M. Falcke, L. Schimansky-Geier, and M. A. Zaks, Non-Markovian Approach to Globally Coupled Excitable Systems, Phys. Rev. E 76, 011118 (2007).

[37] B. Connors and M. Long, Electrical Synapses in the Mammalian Brain, Annu. Rev. Neurosci. 27, 393 (2004).

[38] B. Connors, Synchrony and So Much More: Diverse Roles for Electrical Synapses in Neural Circuits, Dev. Neurobiol. 77, 610 (2017).

[39] P. Coulon and C. Landisman, The Potential Role of Gap Junctional Plasticity in the Regulation of State, Neuron 93, 1275 (2017).

[40] P. Alcami and A. Pereda, Beyond Plasticity: The Dynamic Impact of Electrical Synapses on Neural Circuits, Nat. Rev. Neurosci. 20, 253 (2019).

[41] Electrical synapses are widely observed throughout the mammalian and invertebrate nervous system [42], but may be less frequent than chemical synapses in some neural networks. This choice of coupling simplifies our theoretical derivations. The phenomena reported are, however, more general than neural networks with electrical coupling, and when exploring universality, we show that confining coupling (those couplings promoting coherence in the state variables) leads to synchronization, and such couplings include, in particular, neural networks with chemical coupling and excitatory and inhibitory populations.

[42] M. V. Bennett and R.S. Zukin, Electrical Coupling and Neuronal Synchronization in the Mammalian Brain, Neuron 41, 495 (2004).

[43] C. Hammond, H. Bergman, and P. Brown, Pathological Synchronization in Parkinson's Disease: Networks, Models and Treatments, Trends Neurosci. 30, 357 (2007).

[44] M. McGregor and A. Nelson, Circuit Mechanisms of Parkinson's Disease, Neuron 101, 1042 (2019).

[45] P. Payoux, P. Remy, P. Damier, M. Miloudi, I. Loubinoux, B. Pidoux, V. Gaura, O. Rascol, Y. Samson, and Y. Agid, Subthalamic Nucleus stimulation Reduces Abnormal Motor Cortical Overactivity in Parkinson Disease, Arch. Neurol. 61, 1307 (2004).
[46] R. Chen, S. Kumar, R. Garg, and A. Lang, Impairment of Motor Cortex Activation and Deactivation in Parkinson's Disease, Clin. Neurophysiol. 112, 600 (2001).

[47] B. Degos, J.-M. Deniau, M. Chavez, and N. Maurice, Subthalamic Nucleus High-Frequency Stimulation Restores Altered Electrophysiological Properties of Cortical Neurons in Parkinsonian Rat, PLoS One 8, e83608 (2013).

[48] B. C. Schwab, T. Heida, Y. Zhao, S. A. van Gils, and R. J. van Wezel, Pallidal Gap Junctions-Triggers of Synchrony in Parkinson's Disease?, Mov. Disord. 29, 1486 (2014).

[49] S. Phookan, A. C. Sutton, I. Walling, A. Smith, K. A. O'Connor, J. C. Campbell, M. Calos, W. Yu, J. G. Pilitsis, J. M. Brotchie et al., Gap Junction Blockers Attenuate Beta Oscillations and Improve Forelimb Function in Hemiparkinsonian Rats, Exp. Neurol. 265, 160 (2015).

[50] A.-L. Benabid, P. Pollak, A. Louveau, S. Henry, and J. De Rougemont, Combined (Thalamotomy and Stimulation) Stereotactic Surgery of the VIM Thalamic Nucleus for Bilateral Parkinson Disease, Stereotact. Funct. Neurosurg. 50, 344 (1987).

[51] J. S. Perlmutter and J. W. Mink, Deep Brain Stimulation, Annu. Rev. Neurosci. 29, 229 (2006).

[52] P. Bain, Deep Brain Stimulation (Oxford University Press, New York, 2009).

[53] P. Limousin, P. Pollak, A. Benazzouz, D. Hoffmann, J. Le Bas, E. Broussolle, J. Perret, and A. Benabid, Effect of Parkinsonian Signs and Symptoms of Bilateral Subthalamic Nucleus Stimulation, Lancet 345, 91 (1995).

[54] K. Ashkan, P. Rogers, H. Bergman, and I. Ughratdar, Insights into the Mechanisms of Deep Brain Stimulation, Nat. Rev. Neurol. 13, 548 (2017).

[55] R. Kumar, A. Lozano, Y. Kim, W. Hutchison, E. Sime, E. Halket, and A. Lang, Double-Blind Evaluation of Subthalamic Nucleus Deep Brain Stimulation in Advanced Parkinson's Disease, Neurology 51, 850 (1998).

[56] M. Rodriguez-Oroz, J. Obeso, A. Lang, J.-L. Houeto, P. Pollak, S. Rehncrona, J. Kulisevsky, A. Albanese, J. Volkmann, M. Hariz et al., Bilateral Deep Brain Stimulation in Parkinson's Disease: A Multicentre Study with 4 Years Follow-Up, Brain 128, 2240 (2005).

[57] D. Aum and T. Tierney, Deep Brain Stimulation: Foundations and Future Trends., Front. Biosci. 23, 162 (2018).

[58] T. Heida and J. Modolo, Models of Deep Brain Stimulation, Scholarpedia 12, 33311 (2017).

[59] J. E. Rubin and D. Terman, High Frequency Stimulation of the Subthalamic Nucleus Eliminates Pathological Thalamic Rhythmicity in a Computational Model, J. Comput. Neurosci. 16, 211 (2004).

[60] S. Breit, J. B. Schulz, and A.-L. Benabid, Deep Brain Stimulation, Cell Tissue Res. 318, 275 (2004).

[61] T. Heida, E. Wentink, and J. Geelen, Effectiveness of Deep Brain Stimulation in Subthalamic Nucleus in Parkinson's Disease Somatotopic Organisation, in Proceedings of the 4th European Conference of the International Federation for Medical and Biological Engineering (Springer, Berlin, Heidelberg, 2009), Vol. 22, pp. 59-62.

[62] R. FitzHugh, Mathematical Models of Threshold Phenomena in the Nerve Membrane, Bull. Math. Biol. 17, 257 (1955). 
[63] See Supplemental Material at http://link.aps.org/ supplemental/10.1103/PhysRevX.10.011073 for Movie of the trajectories of 100 neurons out of a 4000 neurons network, depicted in the phase plane $(v, w)$, for each of the 5 parameter set shown in Fig. 1(a). Blue curve: $v$-nullcline, Red curve: $w$-nullcline. Yellow circles: resting neurons, Cyan circles: pioneers.

[64] This distribution is comparable to the distribution of voltage in the uncoupled case, and asymptotically converges to it for vanishing interaction [65].

[65] S. Mischler, C. Quininao, and J. Touboul, On a Kinetic FitzHugh-Nagumo Model of Neuronal Network, Commun. Math. Phys. 342, 1001 (2016).

[66] Noise and coupling indeed have opposite effects. Time rescaling $t^{\prime}=\sigma^{2} t$ indeed leads to a diffusion equation with unit noise variance, leading to an effective coupling coefficient $J / \sigma^{2}$. This rescaling also has an impact on other parameters in the system, including excitability and timescales, so there is no invariance in the system and one cannot assume that the impact of $J$ and $\sigma$ only depend on the ratio $J / \sigma^{2}$.

[67] In the extreme case of coupling diverging to infinity, it can be shown that the distribution of state variables converges to a Dirac mass centered at a time-varying point whose dynamics follow the FitzHugh-Nagumo equation [30], thus, after some time, accumulating at the resting state. Moreover, in that study, a Gaussian profile is predicted for large (but not infinite) $J$, opening the way in that regime to apply Gaussian moment closure as in Ref. [33].

[68] Note that this significant bimodality precludes generalizing the Gaussian approximation of Ref. [33] to nonsmall noise levels; in particular, using the moment ODE for such parameters yields very distinct behaviors with no oscillation, arguing for the importance of bimodality in the model.

[69] Note also that neglecting the evolution of the recovery variable has little impact on the collective dynamics since electrical coupling only relies on voltage.

[70] If a single equilibrium exists, then the system cannot support a mixture of pioneers and resting neurons, and no critical value of $\alpha$ exists, as, regardless of the initial proportion of pioneers, all neurons eventually reach the same equilibrium; if that equilibrium is on the pioneer's branch, a spike will be fired regardless of $\alpha$, while if the equilibrium is on the resting state, no chain reaction occurs.

[71] M. Freidlin and A. Wentzell, Random Perturbations of Dynamical Systems (Springer-Verlag, Berlin, 1998).

[72] H. A. Kramers, Brownian Motion in a Field of Force and the Diffusion Model of Chemical Reactions, Physica (Amsterdam) 7, 284 (1940).

[73] Quantitative estimates of $w_{0}$ as a function of those parameters is a complex task, owing to the fact that relevant noise levels are nonvanishing and to the collective nature of the stabilization. These aspects prevent us from readily extending methods used in the small noise limit for single particles. For a single particle and in the scaling regime of SISR, DeVille et al. [10] estimated the dependence of $w_{0}$ in $\sigma$. In detail, for a single neuron with small noise and recovery timescale with a specific scaling $\left[\sigma^{2} \log \left(\varepsilon^{-1}\right)\right.$ of order 1], an analytic approach allows quantifying $w_{0}$ as a function of $\sigma$ using stochastic estimates combining the relative values of exponential attraction toward the equilibrium invariant manifold (leftmost or rightmost branch of the cubic nullcline) and the exponential switching rate across the quasiseparatrix obtained by Freidlin-Wentzell estimates. These methods fail when noise is nonvanishing, as large-deviations estimates are asymptotic results for vanishing noise.

[74] We chose normally distributed initial conditions with a truncation. For pioneers-to-rest (rest-to-pioneer) transition, this initial condition was centered at $v_{p}\left(v_{r}\right)$ and truncated to ensure $v>v_{u}\left(v<v_{u}\right)$, where $\left(v_{r}, v_{u}, v_{p}\right)$ are defined as the solutions of $f(v)=w_{0}$.

[75] The choice of the threshold did not affect qualitatively the results, yet a threshold exactly at $v_{u}$ was prone to detecting false transitions, and our choice of threshold has the advantage of taking into account the distance between the quasiseparatrix and the equilibrium compared to fixed threshold that would underestimate transitions toward the equilibrium closest to the quasiseparatrix.

[76] A. Clauset, C. R. Shalizi, and M. E. Newman, Power-Law Distributions in Empirical Data, SIAM Rev. 51, 661 (2009).

[77] C. Greene, http://www.chadagreene.com.

[78] J. M. Deniau, B. Degos, C. Bosch, and N. Maurice, Deep Brain Stimulation Mechanisms: Beyond the Concept of Local Functional Inhibition, Eur. J. Neurosci. 32, 1080 (2010).

[79] S. P. Onn and A. A. Grace, Dye Coupling between Rat Striatal Neurons Recorded In Vivo: Compartmental Organization and Modulation by Dopamine, J. Neurophysiol. 71, 1917 (1994).

[80] A. M. Lozano, N. Lipsman, H. Bergman, P. Brown, S. Chabardes, J. W. Chang, K. Matthews, C. C. McIntyre, T. E. Schlaepfer, M. Schulder, Y. Temel, J. Volkmann, and J. K. Krauss, Deep Brain Stimulation: Current Challenges and Future Directions, Nat. Rev. Neurol. 15, 148 (2019).

[81] S. Momin, P. Mahlknecht, D. Georgiev, T. Foltynie, L. Zrinzo, M. Hariz, A. Zacharia, and P. Limousin, Impact of Subthalamic Deep Brain Stimulation Frequency on Upper Limb Motor Function in Parkinson's Disease, J. Parkinsons Dis. 8, 267 (2018).

[82] V. de Carvalho Fagundes, C. R. M. Rieder, A. N. da Cruz, B. C. Beber, and M. W. Portuguez, Deep Brain Stimulation Frequency of the Subthalamic Nucleus Affects Phonemic and Action Fluency in Parkinson's Disease, Parkinsons Dis. (2016) 6760243.

[83] D. Su, H. Chen, W. Hu, Y. Liu, Z. Wang, X. Wang, G. Liu, H. Ma, J. Zhou, and T. Feng, Frequency-Dependent Effects of Subthalamic Deep Brain Stimulation on Motor Symptoms in Parkinson's Disease: A Meta-Analysis of Controlled Trials, Sci. Rep. 8, 14456 (2018).

[84] L. di Biase and A. Fasano, Low-Frequency Deep Brain Stimulation for Parkinson's Disease: Great Expectation or False Hope?, Mov. Disord. 31, 962 (2016).

[85] M. Balaz, M. Bockova, and I. Rektor, Dbs Amplitude Setting Can Improve Aspects of Quality of Life in Patients with Parkinson's Disease, J. Neural. Transm. 120, 643 (2013).

[86] A. Conovaloff, N. Krishnamurthi, P. Mahant, J. Samanta, and J. Abbas, Effects of Deep Brain Stimulation Amplitude 
on Motor Performance in Parkinson's Disease, J. Parkinsonism Restless Legs Syndrome 2, 73 (2012).

[87] J. P. Keener and L. Glass, Global Bifurcations of a Periodically Forced Nonlinear Oscillator, J. Math. Biol. 21, 175 (1984).

[88] J. C. Lilly, Injury and Excitation by Electric Currents, in Electrical Stimulation of the Brain, edited by D. E. Sheer (University of Texas Press for Hogg Foundation for Mental Health, Austin, TX, 1961), Chap. 6, pp. 60-64.

[89] We note that for increasing stimulation frequency, the power spectrum shows complex variations that combine the oscillations-induced desynchronization with more classical phase-locking phenomena.

[90] F. Rieke, D. Warland, R. d. R. Van Steveninck, and W. S. Bialek, Spikes: Exploring the Neural Code (MIT Press Cambridge, MA, 1999), Vol. 7.

[91] M. Dipoppa and B. S. Gutkin, Flexible Frequency Control of Cortical Oscillations Enables Computations Required for Working Memory, Proc. Natl. Acad. Sci. U.S.A. 110, 12828 (2013).

[92] C. Morris and H. Lecar, Voltage Oscillations in the Barnacle Giant Muscle Fiber, Biophys. J. 35, 193 (1981).

[93] E. Marder and J. Goaillard, Variability, Compensation and Homeostasis in Neuron and Network Function, Nat. Rev. Neurosci. 7, 563 (2006).

[94] J. DeFelipe, Neocortical Neuronal Diversity: Chemical Heterogeneity Revealed by Colocalization Studies of Classic Neurotransmitters, Neuropeptides, CalciumBinding Proteins, and Cell Surface Molecules, Cerebral Cortex 3, 273 (1993).

[95] J. M. Tepper, F. Tecuapetla, T. Koós, and O. IbáñezSandoval, Heterogeneity and Diversity of Striatal GABAergic Interneurons, Front. Neuroanat. 4, 150 (2010).

[96] Sensory Neurons: Diversity, Development, and Plasticity, edited by S. A. Scott (Oxford University Press, New York, 1992).

[97] O. Sporns, D. R. Chialvo, M. Kaiser, and C. C. Hilgetag, Organization, Development and Function of Complex Brain Networks, Trends Cognit. Sci. 8, 418 (2004).

[98] A. Arenas, A. Díaz-Guilera, J. Kurths, Y. Moreno, and C. C. Zhou, Synchronization in Complex Networks, Phys. Rep. 469, 93 (2008).

[99] H. Sompolinsky, A. Crisanti, and H. J. Sommers, Chaos in Random Neural Networks, Phys. Rev. Lett. 61, 259 (1988).

[100] G. Hermann and J. D. Touboul, Heterogeneous Connections Induce Oscillations in Large-Scale Networks, Phys. Rev. Lett. 109, 018702 (2012).

[101] E. Meron, Pattern Formation in Excitable Media, Phys. Rep. 218, 1 (1992).

[102] We limited the standard deviation to a value below 1 to keep a significant fraction of neurons with $a^{i}<1$, Avoiding having to switch the roots $a$ and 1 of the cubic function. There is no restriction on the standard deviation of the input.

[103] A. Buchin, S. Rieubland, M. Häusser, B. S. Gutkin, and A. Roth, Inverse Stochastic Resonance in Cerebellar Purkinje Cells, PLoS Comput. Biol. 12, e1005000 (2016).

[104] H. C. Tuckwell and J. Jost, Analysis of Inverse Stochastic Resonance and the Long-Term Firing of HodgkinHuxley Neurons with Gaussian White Noise, Physica (Amsterdam) 391A, 5311 (2012).
[105] A. Ecker, P. Berens, G. Keliris, M. Bethge, N. Logothetis, and A. Tolias, Decorrelated Neuronal Firing in Cortical Microcircuits, Science 327, 584 (2010).

[106] A. Renart, J. De la Rocha, P. Bartho, L. Hollender, N. Parga, A. Reyes, and K. Harris, The Asynchronous State in Cortical Circuits, Science 327, 587 (2010).

[107] A. A. Kühn, F. Kempf, C. Brücke, L. G. Doyle, I. Martinez-Torres, A. Pogosyan, T. Trottenberg, A. Kupsch, G.-H. Schneider, M. I. Hariz, and W. Vandenberghe, High-Frequency Stimulation of the Subthalamic Nucleus Suppresses Oscillatory $\beta$ Activity in Patients with Parkinson's Disease in Parallel with Improvement in Motor Performance, J. Neurosci. 28, 6165 (2008).

[108] M. Vandecasteele, S. Valverde, C. Piette, G. Gangarossa, W. Derousseaux, A. Aristieta Arbelaiz, J. Touboul, B. Degos, and L. Venance, Opto-Activation of Cortical Somatostatin Interneurons Alleviates Parkinsonian Symptoms, https://doi.org/10.1101/460535.

[109] N. Brunel, Dynamics of Sparsely Connected Networks of Excitatory and Inhibitory Spiking Neurons, J. Comput. Neurosci. 8, 183 (2000).

[110] T. P. Vogels and L. F. Abbott, Signal Propagation and Logic Gating in Networks of Integrate-and-Fire Neurons, J. Neurosci. 25, 10786 (2005).

[111] A. Leblois, T. Boraud, W. Meissner, H. Bergman, and D. Hansel, Competition between Feedback Loops Underlies Normal and Pathological Dynamics in the Basal Ganglia, J. Neurosci. 26, 3567 (2006).

[112] P. A. Tass, Phase Resetting in Medicine and Biology: Stochastic Modelling and Data Analysis (Springer-Verlag, Berlin, 2006).

[113] C. Hauptmann and P. A. Tass, Therapeutic Rewiring by Means of Desynchronizing Brain Stimulation, BioSystems 89, 173 (2007).

[114] W. M. Grill, A. N. Snyder, and S. Miocinovic, Deep Brain Stimulation Creates an Informational Lesion of the Stimulated Nucleus, NeuroReport 15, 1137 (2004).

[115] P. A. Tass, Stochastic Phase Resetting: A Theory for Deep Brain Stimulation, Prog. Theor. Phys. Suppl. 139, 301 (2000).

[116] A. T. Winfree, The Geometry of Biological Time (Springer, Berlin, 1980).

[117] A. Priori, G. Foffani, L. Rossi, and S. Marceglia, Adaptive Deep Brain (aDBS) Controlled by Local Field Potential Oscillations, Exp. Neurol. 245, 77 (2013).

[118] M. Goldobin, D. Rosenblum, and A. Pikovsky, Controlling Oscillator Coherence by Delayed Feedback, Phys. Rev. E 67, 061119 (2003).

[119] M. Rosenblum and A. Pikovsky, Delayed Feedback Control of Collective Synchrony: An Approach to Suppression of Pathological Brain Rhythms, Phys. Rev. E 70, 041904 (2004).

[120] P. A. Tass, J. Klosterkötter, F. Schneider, D. Lenartz, A. Koulousakis, and V. Sturm, Obsessive-Compulsive Disorder: Development of Demand-Controlled Deep Brain Stimulation with Methods from Stochastic Phase Resetting, Neuropsychopharmacology : official publication of the American College of Neuropsychopharmacology 28, S27 (2003). 